\title{
An automated method for detection of layer activation order in information processing pathway of rat barrel cortex under mechanical whisker stimulation
}

\author{
Mufti Mahmud $^{\mathrm{a}, \mathrm{b}}$, Elisabetta Pasqualotto ${ }^{\mathrm{a}}$, Alessandra Bertoldo ${ }^{\mathrm{b}}$, Stefano \\ Girardi $^{\mathrm{a}}$, Marta Maschietto ${ }^{\mathrm{a}}$, Stefano Vassanelli ${ }^{*}$ a \\ ${ }^{a}$ NeuroChip Laboratory, Department of Human Anatomy and Physiology, University of \\ Padova, via f. Marzolo 3, 35131 Padova, Italy \\ ${ }^{b}$ Department of Information Engineering, University of Padova, via Gradenigo 6/B, \\ 35131 Padova, Italy
}

\begin{abstract}
Rodents perform object localization, texture and shape discrimination very precisely through whisking. During whisking, microcircuits in corresponding barrel columns get activated to segregate and integrate tactile information through the information processing pathway. Sensory signals are projected through the brainstem and thalamus to the corresponding 'barrel columns' where different cortical layers are activated during signal projection. Therefore, having precise information about the layer activation order is desirable to better understand this signal processing pathway. This work proposes an automated, computationally efficient and easy to implement method to determine the cortical layer activation from intracortically recorded local field potentials (LFPs) and derived current source density (CSD) profiles:

1. Barrel cortex LFPs are represented by a template of four subsequent events: small positive/negative (E1) $\rightarrow$ large negative (E2) $\rightarrow$ slow positive (E3) $\rightarrow$ slow long negative (E4). The method exploits the layer specific characteristics of LFPs to obtain latencies of the individual events (E1-E4), then taking the latency of E2 for calculating the layer activation order.

2. The corresponding CSD profile is calculated from the LFPs and the first sink's peak is considered as a reference point to calculate latencies and evaluate the layer activation order. Other reference points require manual
\end{abstract}

\footnotetext{
*Corresponding author. Tel.: +39 049 8275337; fax: +39 0498275301.

Email address: stefano.vassanelli@unipd.it (Stefano Vassanelli)
} 
calculation.

Similar results of layer activation sequence are found using LFPs and CSDs. Extensive tests on LFPs recorded using standard borosilicate micropipettes demonstrated the method's workability. An interpretation of layer activation order and CSD profiles on the basis of a simplified interacortical barrel column architecture is also provided.

Key words: Layer activation order, barrel cortex, whisker stimulation, local field potentials, current source density.

\section{Introduction}

To explain brain activity underlying perception as the outcome of elementary neuronal responses is one of the major challenges of sensory systems neuroscience. Through "whisking", rodents make extremely fine discriminations of the environment, e.g., object localization, basing on shapes and textures of the objects (Ahissar and Knusten, 2008). The mammal cortex shows a high degree of areal and laminar differentiation and also a representation of sensory surfaces. Especially for the rodents there is a precise topological map of the mystacial pad in the S1 cortex, in which for each whisker there is a so called "barrel" that receives the tactile information (Diamond et al., 2008). Barrels play a very important role in segregation, integration and transmission of sensory information as sensory innervations at each whisker follicle are numerous (larger follicles receives terminations from approximately 200 trigeminal ganglion cells and the smaller follicles about 50) (Fox, 2008). During transmission of the information different layers of the barrel cortex are activated at different times. Studies have shown that intra- and transcolumnar microcircuits in the barrel cortex segregate and integrate information during this activation (Schubert et al., 2007) and that these microcircuits have specific understanding of 'what', 'where' and 'when' aspects of the tactile information acquired by the whiskers.

To have precise knowledge about this information processing pathway by means of extracellular recording and offline signal analysis, an automated, reliable, and quick method is required. Indeed, scientists commonly perform this work manually spending lot of time especially when signals are recorded using neural probes with multiple recording sites.

In this work, we present an automated, simple to implement and computationally efficient method (computational complexity $O\left(n^{2}\right)$ ) capable of 
detecting various events (E1-E4) that characterize the LFPs recorded from different layers of the barrel cortex upon mechanical whisker stimulation. Latencies of the different events from the stimulus-onset are determined and the activation order of the cortical layers is calculated using the latency of E2 (i.e. the highest negative peak).

Generally, however, scientists determine the cortical layer activation order based on the current flow through the cortical layers by calculating the current source density corresponding to the LFPs. Therefore, this analysis is also implemented in the program, thus allowing for automated calculation of the layer activation order from CSDs obtained using the $\delta$-source inverse current source density ( $\delta$-Source iCSD) method (Pettersen et al., 2006). The program first calculates the latency of the first sink's peak from the stimulus-onset of each CSD, then it groups the recordings layerwise and stores the minimum latencies corresponding to each layer in an increasingly ordered list. The layer activation order is determined automatically by taking the minimum latency of each layer. The program was tested on LFPs measured from the rat barrel cortex under whisker stimulation. Resulting CSDs and layer activation order were comparable with previously recorded data (Jellema et al., 2004) and compatible with the intracortical network architecture of the barrel cortex (Fox, 2008). We found that the activation order estimated using the LFPs and CSDs are similar. Also, automated results on layer activation order using LFPs were supported by an in-depth manual analysis of the same data samples.

\section{Signal acquisition}

\subsection{Animal preparation}

Wistar rats were maintained in the Animal Research Facility of the Department of Human Anatomy and Physiology (University of Padova, Italy) under standard environmental conditions.

P30-P40 male rats were anesthetized with an induction mixture of Tiletamine $(2 \mathrm{mg} / 100 \mathrm{~g}$ weight $)$ and Xylazine $(1.4 \mathrm{~g} / 100 \mathrm{~g}$ weight). The anesthesia level was monitored throughout the experiment by testing eye and hind-limb reflexes, respiration and checking the absence of whiskers' spontaneous movements. Whenever necessary, additional doses of Tiletamine (0.5 $\mathrm{mg} / 100 \mathrm{~g}$ weight $)$ and Xylazine $(0.5 \mathrm{~g} / 100 \mathrm{~g}$ weight $)$ were provided.

During the surgery and the recording section, animals were kept on a common stereotaxic apparatus under a stereomicroscope and fixed by teeth 
and ear bars. The body temperature was constantly monitored with a rectal probe and maintained at about $37^{\circ} \mathrm{C}$ using a homeothermic heating pad. Heart beat was assessed by standard ECG. To expose the cortical area of interest, anterior-posterior opening in the skin was made along the medial line of the head, starting from the imaginary eyeline and ending at the neck. While the skin was kept apart using halsted-mosquito hemostats forceps, the connective tissue between skin and skull was gently removed by means of a bone scraper. Thus, the skull over the right hemisphere was drilled to open a window in correspondence of the somatosensory cortex, S1 $(-1 \div-4$ AP, $+4 \div+8 \mathrm{ML}$ ) (Swanson, 2003). Meninges were then carefully cut by means of forceps at coordinates $-2.5 \mathrm{AP},+6 \mathrm{LM}$ for the subsequent insertion of the recording micropipette.

Throughout all surgical operations and recordings, the brain was bathed by a standard Krebs solution (in mM: NaCl-120, $\mathrm{KCl}-1.99, \mathrm{NaHCO}_{3}-25.56$, $\mathrm{KH}_{2} \mathrm{PO}_{4}-136.09, \mathrm{CaCl}_{2}-2, \mathrm{MgSO}_{4}-1.2$, glucose-11), constantly oxygenated and warmed at $37^{\circ} \mathrm{C}$.

At the end of the surgery, contralateral whiskers were trimmed at about $10 \mathrm{~mm}$ from the mystacial pad.

\subsection{Whisker stimulation and recording}

The recording of LFPs from S1 was performed by means of borosilicate micropipettes (1 $\mathrm{M} \Omega$ resistance), filled with Krebs solution. The pipette was fixed to a micromanipulator so that it was $45^{\circ}$ tilted with respect to the vertical axis of the manipulator, thus being inserted perpendicularly to S1 cortex surface. The figure 1 depicts the experimental setup and the stimulus waveform used in driving the stimulator.

LFPs were evoked by single whiskers mechanical stimulation performed with a custom-made speaker that provides dorsal-ventral movements through a connected tube. The speaker was driven by a waveform generator (Agilent 33250A $80 \mathrm{MHz}$, Agilent Technologies) providing $1 \mathrm{~ms}, 10 \mathrm{~V}$ square stimuli with 150 ms delay. Each whisker, starting from the posterior group, was individually inserted into the tube and the corresponding response was checked at $-750 \mu \mathrm{m}$ depth (cortical layer IV), in order to find the most responsive whisker for the selected recording point in the cortex. The so-called "principal whisker" was then chosen for the recording, and the evoked LFPs were recorded from all the cortical layers with a $90 \mu \mathrm{m}$ recording pitch. For each depth, 100 sweeps with $500 \mathrm{~ms}$ duration are recorded at $20 \mathrm{kHz}$ sampling rate. An open source software, 'WinWCP' (Version: 4.1.0) developed by the 
SIPBS, University of Strathclyde, UK (http://spider.science.strath. ac.uk/sipbs/software_ses.htm) was used for recording the signals.

Figure 1: Experimental setup depicting its various components. The arrow on the metal tube connected to the stimulator shows the direction of its movement. Bottom is the stimulus waveform used in driving the speaker, causing dorsal-ventral movement of whisker that is inserted in the metal tube.

\subsection{The signals}

The LFPs recorded from a barrel column of the rat S1 cortex by stimulating the corresponding whisker can be differentiated by their specific characteristics based on the depth or layer they are recorded from. Figure 2 shows a representative depth profile of one of our experiments.

As illustrated in (Ahrens and Kleinfeld, 2004; Kublik, 2004), usually in upper cortical layers (I, II) the signals are expected to have a small positive peak, followed by a main negative peak, a positive peak and a slow negative valley that gradually tends to reach the baseline at the end. In the middle layers (III, IV, and V) the signals are expected to have the main negative peak (without the first small positive peak) followed by a slow positive peak and a slow negative valley tending to reach zero at the end. Deep in the cortex (layer VI), the main negative peak becomes smaller and usually gets divided into two smaller negative peaks, followed by a slow positive peak and then the slow negative valley. These characteristics of the signals can be exploited in automated detection of the layers from the recorded signals.

Figure 2: Depth profile of local field potentials recorded from the E1 barrel column by stimulating the E1 whisker where the different features of the signals can be easily seen. The full depth profile contained equidistant recordings spaced by $90 \mu m$, but for the ease of visualization only representative signals from each layer are shown.

\section{Method}

\subsection{Determining cortical layer activation order directly from LFPs}

This method is implemented using the MATLAB (http://www . mathworks . com) scripting with an easy to use Graphical User Interface (GUI). The figure 3 shows the GUI that encapsulates the implementation for the ease of use of the non-programming background users. The figure 4 shows the flowchart of its basic operational steps (Mahmud et al., 2010a). 
Figure 3: GUI of the layer activation order calculation method using LFPs. This GUI provides an easy way for the non-programming background users to use the method in analyzing their data obtained from experiments.

The method takes the signal files recorded from the rat barrel cortex upon whisker stimulation as input. For each file it calls a module (the flowchart of the module is shown in figure 5) capable of detecting the events present in that signal and calculating the latencies from the starting of the evoked response.

Figure 4: Flowchart showing the operational steps of the layer activation detection method using LFPs.

The layer of recording is determined basing on a priori information about the recording depths of the LFPs. Finally the activation order of different cortical layers in the barrel column is determined by sorting the layerwise minimum latencies of the second event (E2).

The flowchart of the module used in detection of events and calculation of latencies is shown in figure 5. In this module, firstly the signal is low-pass filtered with $250 \mathrm{~Hz}$ cutoff frequency and is translated by setting the signal amplitude at the stimulus-onset to zero. This translation helps in avoiding the slow deviation of signal that might obscure the real amplitude of the events. The calculation of the latencies is based on the detection of various signal events (see Sec. 2.3) by calculating signal derivatives. A major change in the derivative is used in detecting an event.

Figure 5: Flowchart of the event detection and latency calculation module.

The event detection starts with the detection of the response-onset, which is considered as the starting point of the evoked response. To detect the exact response-onset, the standard deviation of the signal's steady-state (the signal before the stimulus-onset) is calculated. The signal from the stimulus-onset to the next $10 \mathrm{~ms}$ is divided into very small parts (0.5 ms duration), and derivatives of these parts are calculated. The response-onset is the time instance of the signal when a small part's derivative is found to exceed \pm standard deviation of the steady-state.

The events are time locked, which means that a change in the signal derivative in a particular time window (either from up to down or vice versa) 
denotes a particular event. Thus, dividing the signal (from the responseonset till the end of the signal) into smaller parts and then scanning for change of derivative is used in detecting event occurrences.

Special care is taken in case of the E1, which may or may not be present in a signal and if present, may have either positive or negative direction. In case of the positive E1, a threshold of $10 \mu \mathrm{V}$ is set to make sure that it indeed is an event and not just background spontaneous brain activity. If the signal is found to be going down, then the maximum negative peak is found and from this peak the signal ranging $\pm 5 \mathrm{~ms}$ is scanned for occurrence of yet another negative peak. If this second negative peak is found, the E1 is set as the first occurring negative peak and the E2 is the second negative peak, otherwise, the E1 is absent and the E2 is the maximum negative peak.

The detection of the rest of the events is very straightforward. It has been empirically found that the next event (E3, i.e., slow positive peak) occurs within the next $100 \mathrm{~ms}$ of the second event and the last event (E4, i.e., slow negative valley) within the $200 \mathrm{~ms}$ of the previous event.

Once the events (E1-E4) are detected, latencies are calculated by subtracting the occurrence time of these events from the stimulus-onset time. The signal characteristics and the latencies are saved in a file for further processing.

After the latencies are calculated for all signal files, they are assigned to the cortical layers from where the signals were recorded from basing on a priori position information. Minimum latencies associated to each layer are then found and sorted in ascending order to determine the order of cortical layers activation.

\subsection{Determining cortical layer activation order using CSD}

Due to the widespread use of current source density (CSD) analysis to obtain the layer activation order, we implemented also this approach in our program. To calculate the CSDs, we considered the $\delta$-Source Inverse CSD method ( $\delta$-source iCSD) as explained in the next subsection. Figure 6 shows the MATLAB graphical user interface that generates the CSD profile from the LFPs and calculates the layer activation order.

Figure 6: GUI of the layer activation order calculation method using CSDs. 


\subsubsection{The $\delta$-Source iCSD method}

The method, which has been adopted from Pettersen et al. (Pettersen et al., 2006), divides the cortex to infinitely thin current discs each of radius $R$ with constant planar CSD, $C_{p}$. For every recording site there is a disc with a determined $C_{p}$ that lies in the $x y$ plane. In this way, we have a $\delta$ function in the $z$-direction, whose value is $C_{p}$ at the recording site and zero between two consecutive recording sites. The potential $\phi(z)$ at the center of a disc positioned at the position $z^{\prime}$ with recording pitch of $h$ is given by:

$$
\phi\left(z, z^{\prime}\right)=\frac{h}{2 \sigma}\left(\sqrt{\left(z-z^{\prime}\right)^{2}+R^{2}}-\left|z-z^{\prime}\right|\right) C
$$

where $C=C_{p} / h$ is the equivalent volume CSD, that corresponds to the CSD obtained if the planar current was distributed in a box of height $h$ in which the disc is embedded and $\sigma$ is the conductivity tensor in the rat brain (default value is considered as $0.42 \mathrm{~S} / \mathrm{m}$ as experimentally reported by Sekino and Ohsaki, 2009). It is assumed that potential at position $z_{j}$ is due to the sum of contributions from the various discs positioned at the recording electrode's contact points, and can be calculated using:

$$
\begin{aligned}
\phi\left(z_{j}\right) & =\sum_{N}^{i=1}\left[\frac{h}{2 \sigma}\left(\sqrt{\left(z_{j}-z_{i}\right)^{2}+R^{2}}-\left|z_{j}-z_{i}\right|\right)\right] C\left(z_{i}\right) \\
& =\sum_{N}^{i=1} F_{j i} C\left(z_{i}\right)
\end{aligned}
$$

where $F$ is an $N \times N$ matrix, denoting that from $N$ values of LFPs we obtain $N$ values of CSD. Furthermore, the elements of $F$ are affected by the appropriate value of the current disc's radius $(R)$ (experimentally reported values are: $300 \mu \mathrm{m}$ and $200 \mu \mathrm{m}$ by Brett-Green et al., 2001 and Alloway, 2008 , respectively; default value for the method is considered to be $250 \mu \mathrm{m}$ ) and are given by:

$$
F_{j i}=\frac{h}{2 \sigma}\left(\sqrt{\left(z_{j}-z_{i}\right)^{2}+R^{2}}-\left|z_{j}-z_{i}\right|\right)
$$

Now the CSD profiles can be estimated by inversing the matrix $F$ and multiplying with the calculated potentials at various recording positions:

$$
\hat{C}=F^{-1} \phi
$$




\subsubsection{Preprocessing}

Before applying the CSD analysis, raw LFPs are low-pass filtered using a Butterworth filter with cutoff frequency of $250 \mathrm{~Hz}$. This is done to remove the high frequency components present in the LFPs that would cause unexpected oscillations in the calculated CSD profile. Moreover, resolution of CSD calculation depends on the recording pitch, with resolution improving by reducing the pitch. Therefore, to facilitate calculation of CSDs with large recording pitches, often interpolation of LFPs is performed (Rappelsberger et al., 1981). During higher order interpolation high spatial frequency noise occurs especially in the neighborhood of the boundary points. To reduce this high spatial frequency noise a symmetrical, weighted average of the LFP about a given point is applied with the form of equation 6 (Hamming filter) (Szymanski et al., 2009; Ulbert et al., 2001).

For signals recorded using neural probes with multiple recording sites separated by a small pitch (simultaneous recording producing a depth profile) the Hamming filter is not necessary. However, it may be applied for removing spatial noise (if any) present in the recordings. In situations where recording is done at different times at different sites (using micropipettes or any other extracellular electrode), Hamming filtering is applied under the assumption that the physiological response of the animal and the stimulus do not change during the recording session, i.e., the response to the stimulus at a specific depth is reproducible over the whole experiment.

$$
\phi(z)=0.23 \phi(z-h)+0.54 \phi(z)+0.23 \phi(z+h)
$$

After applying the Hamming filter only $N-2$ interior recordings can be considered excluding the first and last recordings.

\subsubsection{Detection of layer activation order using CSD}

After the CSD profile is computed, the sources and sinks for the individual recording site can easily be viewed. The calculation of the sinks' latencies is done by subtracting the time instance of the stimulus-onset from the time instance of the peak of the first sink.

Once the latencies are calculated for the whole CSD profile, recordings for each layer are grouped together and the minimum latencies are selected. To determine the layer activation order, minimum latencies are sorted in ascending order and assigned to the different layers depending on recording depths known a priori. Latencies can be used to gain information on signal propagation within intracortical networks. Representative LFPs recorded from 
the rat barrel cortex under whisker stimulation and corresponding CSDs computed by the program are shown as an example in figure 7 (A, B). Hypothetical signal propagation pathways across the barrel intracortical network are inferred from the CSD profile and the latencies temporal pattern (as seen in figure 7 (C)) (Fox, 2008; Jellema et al., 2004).

To verify the activation order, sinks are shaded and along with sources they are annotated (as seen in figure 7 (B)), and a plausible neuronal network architecture corresponding to the CSD profile is drawn in figure $7(\mathrm{C})$ for explanatory purpose. This hypothetical network architecture simply reflects the current flows that are seen by the sinks and sources in the calculated CSD profile.

Latencies indicated that the signals propagated through a pathway starting from $\mathrm{Vb}$ and then traversing through Va, III, IV, II, I and to VI. This order of layer activation is supported by the known neuronal architecture of the barrel cortical layers. This architecture suggests that the thalamic inputs (VPm) activate the layer $\mathrm{Vb} / \mathrm{IV}$, signals propagate through layer III and II from where the outputs are projected to layer Va, Vb. From these layers the output is sent to the layer VI and then back to the thalamus. 
Figure 7: (A): Depth profile of recorded LFPs. (B): The respective CSD profile computed using $\delta$-source iCSD from the LFPs. The hatched portions of the profile denote the sinks $(\mathrm{a}-\mathrm{l})$ and the negative portions the sources (1-10). Stars indicate the initiation sites of the current flow within the cortex. (C): Barrel column architecture derived from previous studies (Fox, 2008; Jellema et al., 2004) showing the possible connections among neurons in different cortical layers. Arrows indicate either signal propagation (alphabet-to-alphabet: propagation of sinks; number-to-number: propagation of sources) or directed inward current (number-to-alphabet: current flow from source to sink) according to the nomenclature adapted by (Jellema et al., 2004). From the analysis of the CSD profile and latencies it is inferred that, there are two dominant sink-source complexes. The first one is initiated at the upper part of layer Va (sink ' $a$ ') and ending at layer I (sink ' $\mathrm{f}$ '); the second one is initiated at the lower part of layer Vb (sink ' $\mathrm{g}$ ') and ending at layer VI (sink ' $\mathrm{l}$ '). The two complexes are assumed to be caused by the monosynaptic thalamic input (Fox, 2008) and are initiated through the sinks ' $\mathrm{a}$ ' and ' $\mathrm{g}$ ' after whisker stimulation. The first complex is supposed to be initiated by the pyramidal cells situated in the upper part of layer Va. This complex is propagated through neurons in the layer IV, III, and II. The axons of these cells are projected towards layer III (in case of Va) and layer I (in case of IV, III, and II). Propagation of this complex created huge sinks (sinks ' $a$ ' to ' $\mathrm{f}$ ' in the CSD profile) and sources $(1,4,3$, and 6$)$. The second complex is supposed to be initiated by pyramidal cells situated in the lower part of layer $\mathrm{Vb}$ and propagated through the lower portion of layer Va. During this propagation sinks 'g' to ' $\mathrm{k}$ ' with increasing amplitude are generated. Indeed, the sink ' $\mathrm{g}$ ' at the beginning of propagation is relatively small. Afterwards, sinks increase in amplitude and width possibly due to the fact that pyramidal cells involved in propagation receive other excitatory inputs from layer IV. Sources $2,5,7,8$, and 9 are associated to sinks 'g', 'h', 'i', 'j' 'c' and 'k', respectively. In layer VI and deeper polysynaptic delayed inputs caused additional sinks (sinks ' 1 '). Wires represent schematically excitatory connections. 
Figure 8: Simplified architecture of a barrel column as described in (Fox, 2008). When a whisker is stimulated, the information first goes to the thalamus, and then from the thalamus to the corresponding barrel. As it can be seen from the picture, there are two principal thalamic inputs (VPm) that are in layer IV and at the border between layers $\mathrm{Vb}$ and VI. The thalamic inputs in layer IV activate both excitatory (represented by stellate cells) and inhibitory cells (represented by basket cells). These basket cells provide feedforward perisomatic inhibition from the VPm and feedback inhibition to the excitatory stellate cells. The LTS (low threshold spike) cells do not receive a thalamic input directly, so they are involved only in the feedback inhibition. The excitatory output from here is then projected to layers II/III. It should be noted that there are connections between inhibitory cells, in order to increase inhibition, and between the excitatory cells themselves. From the literature, it is known that stellate cells connect mainly with other stellate cells and pyramidal cells with other pyramidal cells. The excitatory cells of the granular layer (IV) then project to the supragranular layers (II/III). The connections between layer IV and layers II/III are numerous and strong, which may reflect the large amplitude of the sinks in these regions. Even in this case, basket cells provide both feedforward and feedback inhibition as the chandelier cells project to the axon initial segment of pyramidal cells. The excitatory outputs from these pyramidal cells are projected to layers V/VI. The output of the supragranular layers becomes the input for the infragranular layers, i.e., layers II-Va and III-Vb reciprocally connect within a column. Pyramidal cells of layers V and VI can be inhibited by inhibitory cells of same or other layers, such as the Martinotti cells. Sensory information finally reaches layer VI, whose cells are reciprocally connected with layer Vb cells, and from there comes back to the thalamus. From the picture it can be noted that layer IV connects also with layer Va and VI cells. The dash-dot-dashed and dashed lines are the feedback connections that project from the layer Vb back to layer III and from the layer VI back to the inhibitory cells of layer IV, respectively.

A simplified architecture of a barrel column reconstructed from (Fox, 2008) is depicted in figure 8. Signal propagation estimated from CSD analysis matches, to a certain extent, the pathway through the single barrel. Of course, as the dynamics of individual events involved in signal generation and propagation through the network is largely unknown, the predictory potential of this network model regarding circuit activation remains limited.

\subsection{Manual calculation of cortical layer activation}

As a proof of automated detection reliability, the layer activation order was also calculated manually from the LFP profile. Latencies from the stimulus-onset were estimated manually for each event (E1-E4) using a commercial software (clampfit, v.10.0, http://www.moleculardevices . com/Products/Software/Electrophysiology/pCLAMP.html). This software provides time instance and amplitude of the signal's data point where the data-cursor was placed. Thus, the events were pointed manually one-by-one 
and the time instances noted. The latencies of the events were calculated by subtracting the stimulus-onset from the time instance of each event. Again, only E2 latencies were considered for calculation of the layer activation order. Then, E2 latencies were grouped layerwise and the minimum latency in each layer was found. These minimum latencies were then sorted in ascending order to determine the signal propagation among the layers. A comparison between automated and manual detection results is shown in the Results and discussion section.

\section{Results and discussion}

The event detection algorithm described in section 3.1 has a computational complexity of $O\left(n^{2}\right)$. Another method proposed for event detection in LFPs (Bokil et al., 2006) was based on an algorithm with higher computational complexity $O(\log n !)$. Furthermore, algorithms used to detect PQRST complexes in ECG signals (Dota et al., 2002, 2009; Piotrowskia and Rozanowski, 2010) can be adapted to detect the events present in LFPs; however, these algorithms have computational complexities of $O\left(n^{3}\right)$.

\subsection{Single experiment}

The method was applied to a number of datasets and found to be working quite well except a few situations ( $2 \%$ of occurrence rate) where an error of $\pm 300 \mu$ s was noticed in latency calculation. Particularly, latency calculation error was occurring in case of signals containing slow stimulus artifacts (with frequency components less than $250 \mathrm{~Hz}$ ). As latencies are in terms of a few milliseconds up to hundred of milliseconds, this error can be considered negligible. Figure 9 shows representative signals and their respective detected events after a run of the method.

Figure 9: LFP depth profile with detected events using the method mentioned in section 3.1. The signals were recorded equidistantly (90 $\mu \mathrm{m}$ pitch). For better visualization only representative signals from each layer are shown.

When compared, the latency results for the layer activation order obtained from the LFPs and the CSD profile (figure 10 and figure 11) are found similar in terms of activation sequence, but not with respect to their values. The layerwise latencies of CSDs are larger than those of the LFPs. This is 
due to the fact that, in case of the CSDs, latencies are calculated as the difference between time instance of the first sink's peak and the stimulus-onset, whereas the latencies for the LFPs are calculated as the difference between the time instance of the E2 and the stimulus-onset.

Figure 10: Comparison of layer-wise latencies calculated from the LFPs and CSDs.

Figure 11: Layer activation order calculated using the LFP (top) and CSD profiles (bottom).

For determining the layer activation order using CSDs, a common reference point is required to calculate the latencies, which can be the exact initiation of the first sink (Kaur et al., 2005; Mitzdorf and Singer, 1980; Mitzdorf, 1985; Swadlow et al., 2002) or the peak of the first sink (CastroAlmancos and Oldford, 2002; Di et al., 1990; Megevand et al., 2009; Staba et al., 2004) or a combination of both (Jellema et al., 2004; Szymanski et al., 2009). Due to the oscillations in the CSDs caused by calculation, it is difficult to detect the exact initiation of the first sink in each CSD using an automated method. Indeed, especially when the LFPs contain high spontaneous brain activity or oscillations the generated CSDs are too noisy and oscillatory. Filtering the LFPs or the CSDs does not really eliminate possibility of miscalculation. Therefore, the difficulty in detecting the first sink's onset led us to consider the first sink's peak in calculating the latencies. Scientists need to perform the latency calculation manually in cases where a different reference point is required other than the first sink's peak. To this respect the method suffers a shortcoming which will require a more complex algorithm to overcome. 
Table 1: Comparison of manual and automatic calculation of latencies

\begin{tabular}{cccccc}
\hline \hline \multirow{2}{*}{ Depth } & \multirow{2}{*}{ Mode } & \multirow{4}{c}{ Latencies (ms) } \\
& & E1 & E2 & E3 & E4 \\
\hline \hline \multirow{2}{*}{$90 \mu \mathrm{m}$} & M & 5.384 & 19.784 & 42.934 & 144.954 \\
& A & 5.655 & 19.564 & 42.742 & 143.393 \\
\hline \multirow{2}{*}{$180 \mu \mathrm{m}$} & M & Absent & 19.745 & 60.055 & 174.215 \\
& A & Absent & 19.416 & 59.259 & 174.023 \\
\hline \multirow{2}{*}{$270 \mu \mathrm{m}$} & M & Absent & 19.905 & 64.795 & 180.965 \\
& A & Absent & 19.615 & 63.513 & 183.733 \\
\hline \multirow{2}{*}{$450 \mu \mathrm{m}$} & M & Absent & 20.215 & 69.395 & 232.835 \\
& A & Absent & 20.228 & 70.320 & 232.836 \\
\hline \multirow{2}{*}{$540 \mu \mathrm{m}$} & M & Absent & 20.075 & 74.205 & 221.595 \\
& A & Absent & 20.216 & 74.124 & 222.021 \\
\hline \multirow{2}{*}{$720 \mu \mathrm{m}$} & M & Absent & 20.645 & 79.895 & 283.305 \\
& A & Absent & 20.565 & 78.228 & 282.532 \\
\hline \multirow{2}{*}{$990 \mu \mathrm{m}$} & M & Absent & 19.375 & 87.805 & 220.125 \\
& A & Absent & 19.464 & 87.887 & 175.475 \\
\hline \multirow{2}{*}{$1260 \mu \mathrm{m}$} & M & Absent & 18.585 & 96.025 & 238.595 \\
& A & Absent & 18.213 & 96.046 & 239.489 \\
\hline \multirow{2}{*}{$1620 \mu \mathrm{m}$} & M & 16.1150 & 38.925 & 110.835 & 202.635 \\
& A & 16.116 & 38.785 & 112.562 & 198.448 \\
\hline \multirow{2}{*}{$1800 \mu \mathrm{m}$} & M & 10.175 & 38.585 & 118.825 & 234.975 \\
& A & 10.310 & 38.584 & 118.568 & 234.784 \\
\hline \hline
\end{tabular}

The latencies calculated by the automated method for the LFPs (depth profile can be seen in figure 9) were also compared with the manually calculated latencies and the results were found to be similar (table 1). 'M' denotes manual computation by hand and 'A' denotes automated calculation using the method. In table 1 the 'E1', 'E2', 'E3' and 'E4' are the latencies of the respective events. As mentioned in section 2.3 in the upper layers we can observe the first positive peak, which gradually disappears in the middle layers and eventually becomes the first negative peak. This phenomena is also evident in the tables as the latencies of the E1 in the middle layers is reported 'Absent'. Furthermore, table 2 reports average latencies for 3 different experiments evaluated manually and by the program with their root mean square errors (RMSE). In table 2 the 'E1', 'E2', 'E3' and 'E4' are averaged latencies and RMSE of the respective events. The low RMSE indicates that the calculation of latencies using the automated method is accurate. The tables report data corresponding to representative signal(s) from depth(s) of each layer (please see figure 9). 
Table 2: Average latencies of events using manual and automatic calculation with RMSE

\begin{tabular}{|c|c|c|c|c|c|c|c|c|c|}
\hline \multirow{2}{*}{ Depth } & \multirow{2}{*}{ Mode } & \multicolumn{4}{|c|}{ Average Latencies (ms) } & \multicolumn{4}{|c|}{ RMS Errors } \\
\hline & & E1 & E2 & E3 & $\mathrm{E} 4$ & E1 & $\mathrm{E} 2$ & E3 & $\mathrm{E} 4$ \\
\hline \multirow{2}{*}{$90 \mu \mathrm{m}$} & $\bar{M}$ & 6.019 & 19.784 & 42.450 & $\overline{139.014}$ & \multirow{2}{*}{0.542} & \multirow{2}{*}{0.081} & \multirow{2}{*}{0.024} & \multirow{2}{*}{0.315} \\
\hline & A & 6.592 & 19.564 & 42.201 & 140.047 & & & & \\
\hline \multirow{2}{*}{$180 \mu \mathrm{m}$} & $\mathrm{M}$ & Absent & 19.745 & 67.547 & 178.850 & \multirow{2}{*}{ Absent } & \multirow{2}{*}{0.092} & \multirow{2}{*}{0.021} & \multirow{2}{*}{0.221} \\
\hline & A & Absent & 19.416 & 68.974 & 175.654 & & & & \\
\hline \multirow{2}{*}{$270 \mu \mathrm{m}$} & $\mathrm{M}$ & Absent & 28.517 & 62.574 & 183.015 & \multirow{2}{*}{ Absent } & \multirow{2}{*}{0.026} & \multirow{2}{*}{0.032} & \multirow{2}{*}{0.254} \\
\hline & A & Absent & 28.428 & 65.051 & 187.373 & & & & \\
\hline \multirow{2}{*}{$450 \mu \mathrm{m}$} & $\bar{M}$ & Absent & 25.591 & 74.102 & 221.301 & \multirow{2}{*}{ Absent } & \multirow{2}{*}{0.062} & \multirow{2}{*}{0.028} & \multirow{2}{*}{0.253} \\
\hline & $\mathrm{A}$ & Absent & 25.675 & 77.108 & 203.952 & & & & \\
\hline \multirow{2}{*}{$540 \mu \mathrm{m}$} & $\mathrm{M}$ & Absent & 18.175 & 71.214 & 231.595 & \multirow{2}{*}{ Absent } & \multirow{2}{*}{0.059} & \multirow{2}{*}{0.046} & \multirow{2}{*}{0.477} \\
\hline & A & Absent & 18.318 & 72.980 & 213.741 & & & & \\
\hline \multirow{2}{*}{$720 \mu \mathrm{m}$} & $\mathrm{M}$ & Absent & 20.145 & 72.985 & 210.745 & \multirow{2}{*}{ Absent } & \multirow{2}{*}{0.048} & \multirow{2}{*}{0.094} & 0.351 \\
\hline & A & Absent & 19.619 & 73.428 & 271.659 & & & & 0.001 \\
\hline $990 \mathrm{um}$ & $\mathrm{M}$ & Absent & 21.937 & 84.862 & 192.251 & Absent & 0.095 & 0.392 & 0.853 \\
\hline s.optit & A & Absent & 22.121 & 90.957 & 183.241 & & & & \\
\hline $1260 \mu \mathrm{m}$ & $\mathrm{M}$ & Absent & 18.985 & 91.213 & 210.021 & Absent & 0.036 & 0.095 & 0.764 \\
\hline & A & Absent & 19.018 & 91.478 & 228.674 & & & & \\
\hline $1620 \mu \mathrm{m}$ & $\mathrm{M}$ & 11.152 & 26.132 & 110.835 & 192.380 & 0.152 & 0.071 & 0.93 & 0.429 \\
\hline 1020 קнसн & A & 10.920 & 25.925 & 112.562 & 181.154 & & & & \\
\hline $1800 \mu \mathrm{m}$ & $\mathrm{M}$ & 9.631 & 35.585 & 117.241 & 221.341 & 0.821 & 0.087 & 0.034 & 0.762 \\
\hline & A & 9.927 & 35.885 & 113.231 & 214.114 & & & & \\
\hline
\end{tabular}

Also, activation orders of cortical layers based on manual calculation and by the method are exactly the same (figure 12). Therefore, results of table 1 , 2 and figure 12 suggest that the automated method is accurate in detecting the various events present in the LFPs and in calculating latencies with precisions that are sufficient for a reliable determination of the activation order of cortical layers.

Figure 12: Comparison of manual and automatic method's latency calculation in finding the activation order of different cortical layers.

In conclusion, basing on these evidences, we can assert that the automated method presented can calculate the activation order of layers in the barrel columns upon mechanical whisker stimulation in a single experiment. Both the approaches (using LFP or CSD) provide similar results; it is the choice of the user to decide which one to use based on the need.

\subsection{Average across experiments}

In addition to the single experiments we also applied our method to the grand average across three different experiments. This type of averaging technique has been adopted by previous studies to determine the temporal order of layer activation in the cortex (Di et al., 1990; Jellema et al., 2004; Staba et al., 2004). During these experiments, the signals are recorded at a 
pitch of $90 \mu \mathrm{m}$ by mechanically stimulating the D1 whisker. Thus, the signals were averaged across experiments (depthwise) to obtain a grand average LFP profile. This profile was then used to calculate the layer activation order using LFPs and CSDs.

To obtain the layer activation order from the grand average LFPs, the latencies of different events (E1-E4) were calculated and grouped layerwise. The minimum latency in each layer was found and sorted in ascending order, thus providing the activation order. The CSD profile was calculated using the grand average LFP profile through application of $\delta$-iCSD method. Latencies were calculated considering the first sink's peak and were grouped layerwise. The minimum latency in each layer was found and sorted in ascending order to obtain the activation order.

Figure 13 shows the latencies obtained from the grand average LFP profile and the CSD profile obtained using the $\delta$-iCSD method. Standard deviations of the means are shown as vertical bars. The latencies at different depths obtained using the grand average by the two methods show a temporal order of layer excitation comparable to previous studies (Armstrong-James et al., 1992; Di et al., 1990; Einevoll et al., 2007).

Figure 13: Latencies obtained from the grand average $(n=3)$. Latencies calculated using LFP based method (top) and latencies calculated using CSD based method (bottom). The vertical bars show standard deviations of the means.

\section{Conclusion}

Whisking in the rodents is one of the most important ways in exploring the environments. To understand the whisking mechanism, its role in localizing objects and discriminate among them based on shape and texture are under extensive study. To perform this kind of studies, determining the signal processing pathway and, in turn, the order of activation of different cortical layers is very important. Scientists perform this task manually which is time consuming and boring. As evidenced above, the proposed method is an automated solution in performing this kind of analysis. Two methods are automated, one uses the LFPs and the other one the CSDs. Depending on the need, the user can select either of them and have a qualitative assessment of the layer activation order. Moreover, the method using LFPs is computationally efficient, quick and easy to implement. As it involves less calculations compared to the CSD based approach, this method could 
be preferably adapted for analysis of signals recorded using high resolution brain-chip interfaces or neural probes capable of recording large amounts of data during an experiment. This layer activation order detection toolbox is a part of the SigMate software package that will be made available to the community shortly (Mahmud et al., 2010b).

\section{Acknowledgment}

This work was carried out as a part of the European Commission funded CyberRat project under the Seventh Framework Programme (ICT-2007.8.3 Bio-ICT convergence, 216528, CyberRat).

\section{References}

Ahissar E, Knutsen KM. Object localization with whiskers. Biol Cybern 2008; 98(6):449-58.

Ahrens KF, Kleinfeld D. Current flow in vibrissa motor cortex can phase-lock with exploratory rhythmic whisking in rat. J Neurophysiol, 2004; 92:1700-7.

Alloway KD. Information processing streams in rodent barrel cortex: the differential functions of barrel and septal circuits. Cereb Cortex, 2008; 18(5):979-98.

Armstrong-James M, Fox K, Das-Gupta A. Flow of excitation within rat barrel cortex on stiking a single vibrissa. J Neurophysiol 1992; 68(4):1345-57.

Bokil HS, Pesaran B, Andersen RA, Mitra PP. A method for detection and classification of events in neural activity. IEEE T Bio-Med Eng, 2006; 53(8): 1678-87.

Brett-Green BA, Chen-Bee CH, Frostig RD. Comparing the functional representations of central and border whiskers in rat primary somatosensory cortex. J Neurosci, 2001; 21(24):9944-54.

Castro-Alamancos MA, Oldford E. Cortical sensory suppression during arousal is due to the activitydependent depression of thalamocortical synapses. J Physiol, 2002; 541(1): 319-31.

Di S, Baumgartner C, Barth D S. Laminar Analysis of extracellular field potentials in rat vibrissa/barrel cortex. J Neurophysiol, 1990; 63: 832-40.

Diamond ME, vo Heimendahl M, Knutsehn PM, Kleinfeld D, Ahissar D. 'Where' and 'what' in the whisker sensorimotor system. Nat Rev Neurosci, 2008; 9:601-12.

Dota C, Skallefell B, Edvardsson N, Fager G. Computer-based analysis of dynamic QT changes: toward high precision and individual rate correction. Ann Noninvas Electro, 2002; 7(4): 289-301.

Dota CD, Edvardsson N, Skallefell B, Fager G. PC-based ECG waveform recognitionvalidation of novel software against a reference ECG database. Ann Noninvas Electro, 2009; 14(1): S42-7.

Einevoll GT, Pettersen KH, Devor A, Ulbert I, Halgren E, Dale AM. Laminar population analysis: estimating firing rates and evoked synaptic activity from multielectrode recordings in rat barrel cortex. J Neurophysiol 2007; 97:2174-90.

Fox K. Barrel Cortex. Cambridge: Cambridge University Press; 2008. 
Jellema T, Brunia CHM, Wadman WJ. Sequential activation of microcircuits underlying somatosensoryevoked potentials in rat neocortex. Neuroscience, 2004; 129: 283-95.

Kaur S, Rose H J, Lazar R, Liang K, Metherate R. Spectral integration in primary auditory cortex: laminar processing of afferent input, in vivo and in vitro. Neuroscience, 2005; 134: 1033-45.

Kublik E. Contextual impact on sensory processing at the barrel cortex of awake rat. Acta Neurobiol Exp, 2004; 64:229-38.

Mahmud M, Bertoldo A, Maschietto M, Girardi S, Vassanelli S. Automatic detection of layer activation order in information processing pathways of rat barrel cortex under mechanical whisker stimulation. In: Proc. of the 32nd annual international conference of the IEEE engineering in medicine and biology society (IEEE EMBC2010); 2010a. p. 6095-8.

Mahmud M, Bertoldo A, Girardi S, Maschietto M, Vassanelli S. SigMate: a MatLAB-based neuronal signal processing tool. In: Proc. of the 32nd annual international conference of the IEEE engineering in medicine and biology society (IEEE EMBC2010); 2010b. p. 1352-5.

Megevand P, Troncoso E, Quairiaux C, Muller D, Michel CM, Kiss JZ. Long-term plasticity in mouse sensorimotor circuits after rhythmic whisker stimulation. J Neurosc, 2009; 29(16): 5326-35.

Mitzdorf U, Singer W. Monocular activation of visual cortex in normal and monocularly deprived cats: an analysis of evoked potentials. J Physiol, 1980; 304: 203-20.

Mitzdorf U. Current source-density method and application in cat cerebral cortex: investigation of evoked potentials and EEG phenomena. Physiol Rev, 1985; 65: 37-100.

Pettersen KH, Devor A, Ulbert I, Dale AM, Einevoll GT. Current-source density estimation based on inversion of electrostatic forward solution: Effects of finite extent of neuronal activity and conductivity discontinuities. J Neurosci Meth, 2006; 154:116-33.

Piotrowskia Z, Rozanowski K. Robust algorithm for heart rate (HR) detection and heart rate variability (HRV) estimation. Acta Physica Polonica A, 2010; 118(1): 131-5.

Rappelsberger P, Pockberger H, Petsche H. Current source density analysis: methods and application to simultaneously recorded field potentials of the rabbit's visual cortex. Pflugers Arch, 1981; 389:159-70.

Schubert D, Kotter R, Staiger JF. Mapping functional connectivity in barrel-related columns reveals layer- and cell type-specific microcircuits. Brain Struct Funct, 2007; 212:107-19.

Sekino M, Ohsaki H, Yamaguchi-Sekino S, Iriguchi N, Ueno S. Low-frequency conductivity tensor of rat brain tissues inferred from diffusion mri. Bioelectromagnetics, 2009; 30(6):489-99.

Staba RJ, Bergmann PC, Barth DS. Dissociation of slow waves and fast oscillations above $200 \mathrm{~Hz}$ during GABA application in rat somatosensory cortex. J Physiol, 2004; 561(1): 205-14.

Swadlow H A, Gusev A G, Bezdudnaya T. Activation of a cortical column by a thalamocortical impulse. J Neurosci, 2002; 22(17): 7766-73.

Swanson LW. Brain Maps: Structure of the Rat Brain, third ed. London: Academic Press; 2003.

Szymanski FD, Garcia-Lazaro JA, Schnupp JWH. Current source density profiles of stimulus-specific adaptation in rat auditory cortex. J Neurophysiol, 2009; 102:1483-90.

Ulbert I, Karmos G, Heit G, Halgren E. Early discrimination of coherent versus incoherent motion by multiunit and synaptic activity in human putative MT+. Hum Brain Mapp, 2001; 13: 226-38. 


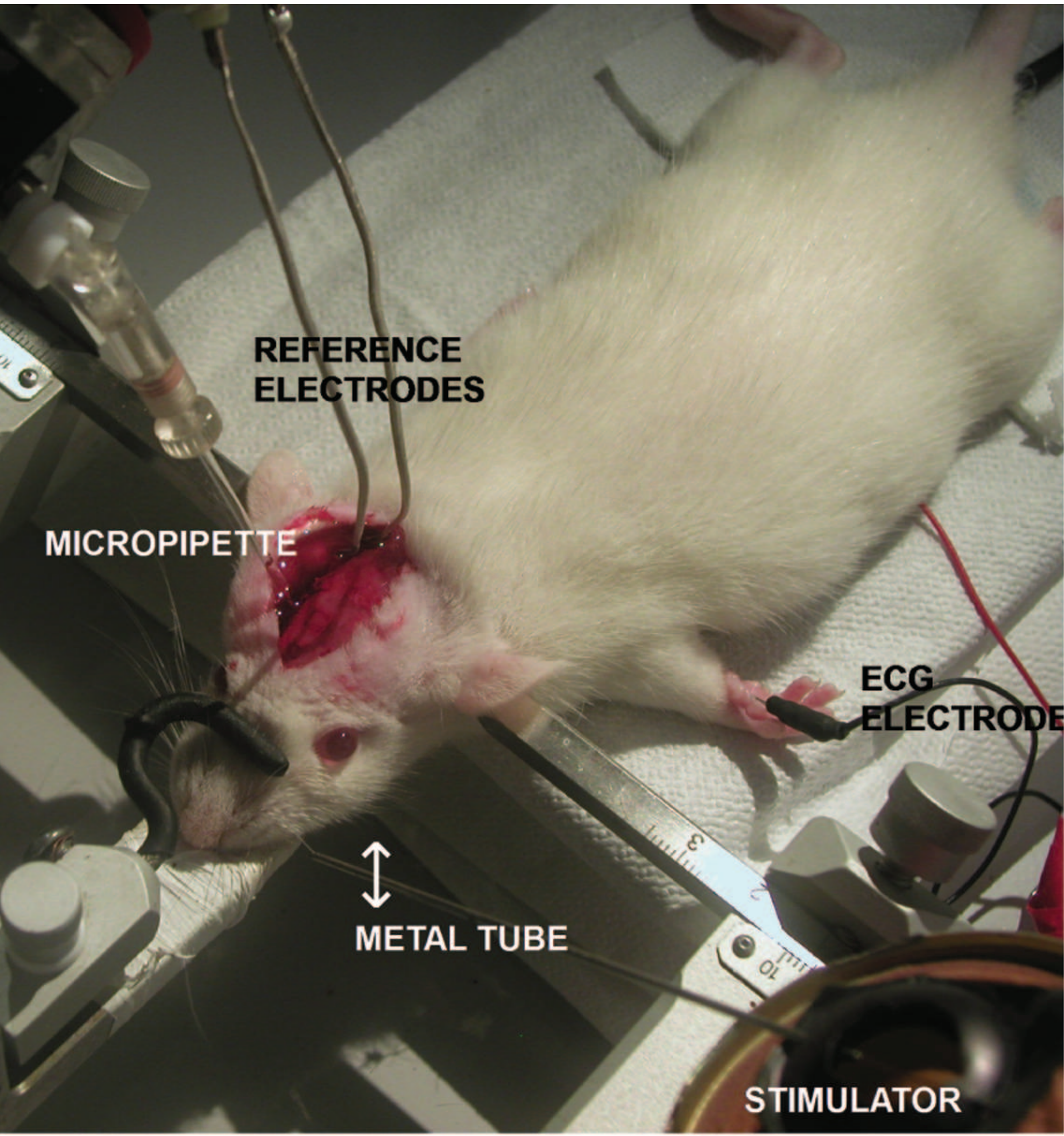

\section{$1 \mathrm{~ms}$}

$D_{\text {One }}$

Mahmud, M., Pasqualotto, E., Bertoldo, A., Girardi, S., Maschietto, M., Vassanelli, S. An automated method for detection er activation order in information processing pathway of rat barrel cortex under mechanical whisker stimulation. (2011)

\section{$150 \mathrm{~ms}$}


Figure 2

\section{Recorded Signal}

\section{Layers}

90

270

360

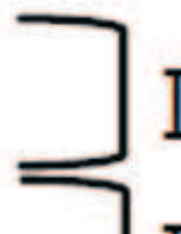

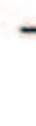

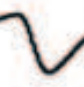

2

$\sqrt{2}$

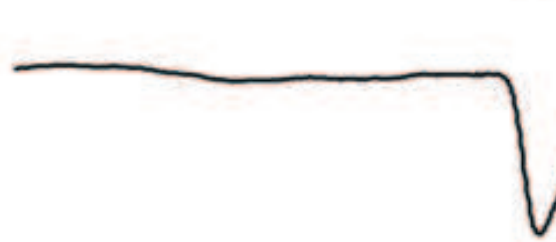

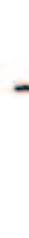

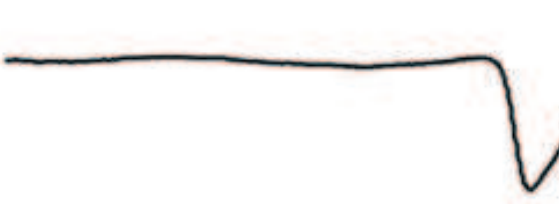

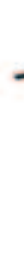
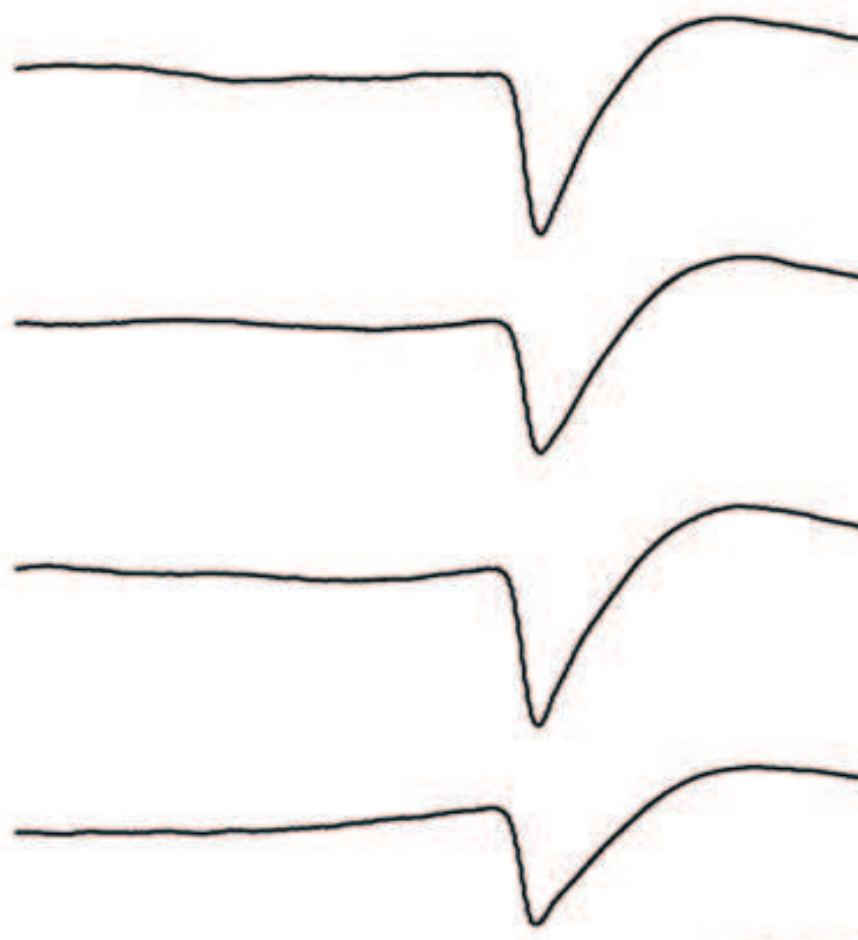

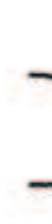
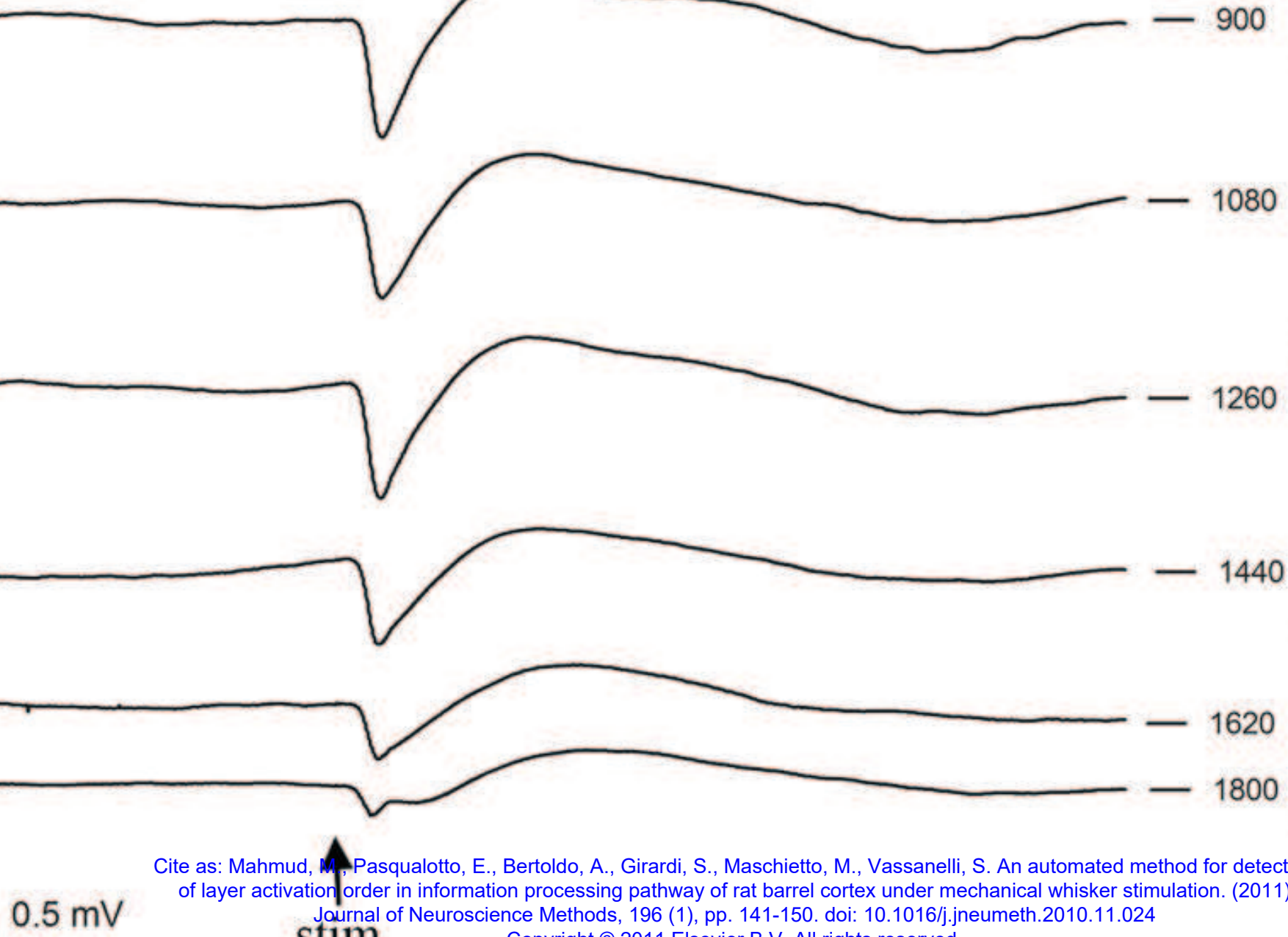

Cite as: Mahmud, Pasqualotto, E., Bertoldo, A., Girardi, S., Maschietto, M., Vassanelli, S. An automated method for detection of layer activation order in information processing pathway of rat barrel cortex under mechanical whisker stimulation. (2011)

\section{$100 \mathrm{~ms}$} Journal of Neuroscience Methods, 196 (1), pp. 141-150. doi: 10.1016/j.jneumeth.2010.11.024 
Figure 3

Sig Mate: Analysis Software for Neuronal Signals - Latency Estimator in LFPs

Latency Estimator in LFPs

Select a Signal Source: Micropipette Recordings *

Select a Directory Containing the * txt Fles of Similar Recordings
Select Channels for Analysis:

$\checkmark$ VmaC $\square$ VmDC Stimulus
Browse

Low Pass Cutoff Frequency.
100

$100 \quad \mathrm{~Hz}$ Plot of Selected Column: 1

\section{Plot of Selected Column: 1}

Selected Directory Path:E Un-Vivo Recordings Pipette Recordings Whisker

Stimb20090715 P37 150ar male-analyzedi20090715-Ne Content of the Directory

E1 $x$ COVOza150 imsiovise *

E1_x0y0z-250_1ms $10 \mathrm{~V} 150$

E1_xoy0z-350_1ms $10 \mathrm{~V} 150$

E1_ $x 0 y 0 z-450 \_1 m s 10 \mathrm{~V} 150$

E1_x0yoz-550_1ms10V150

EI $\times 0$ yoz-650_1 1 ms $10 \mathrm{~V} 150$

E1_xoyoz-750_1 $1 \mathrm{~ms} 10 \mathrm{~V} 150$ :

E1_xoy0z-850_1ms $10 \mathrm{~V} 150$

E1_ $\times 0 y 0 z-950 \_1 m_{1} 10 \mathrm{~V} 150$

E1_x0y0z-1050_ims $10 \mathrm{~V} 15$

E1_x0y0z-1150_ims $10 \mathrm{~V} 15$

E1_xOy0z-1250_ims $10 \mathrm{~V} 15$

E1 XOy0z-1350_1ms $10 \mathrm{~V} 15$

E1_ $\times 0 y 0 z-1450 \_$Ims $10 \mathrm{~V} 15$

E1_x0y0z-1550_ims $10 \mathrm{~V} 15$

E1_x0y0z-1650_ims $10 \mathrm{~V} 15$.

:

Remove File

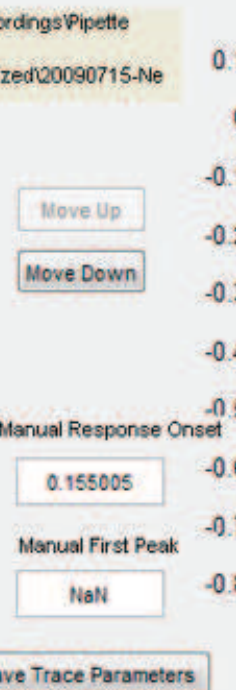

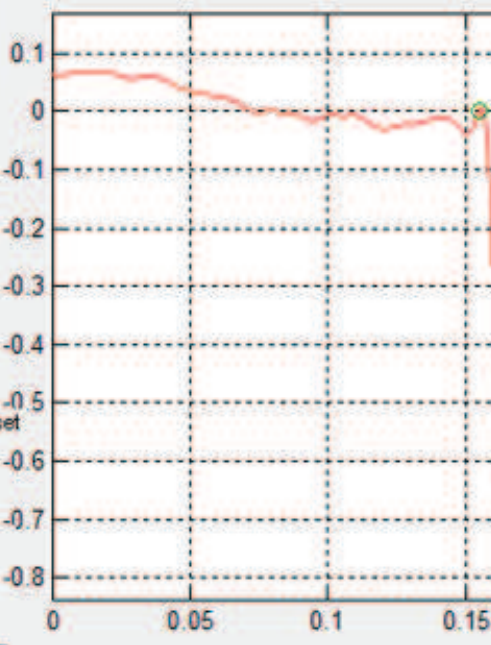

Pan

Data Cursor

Zoom +

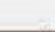
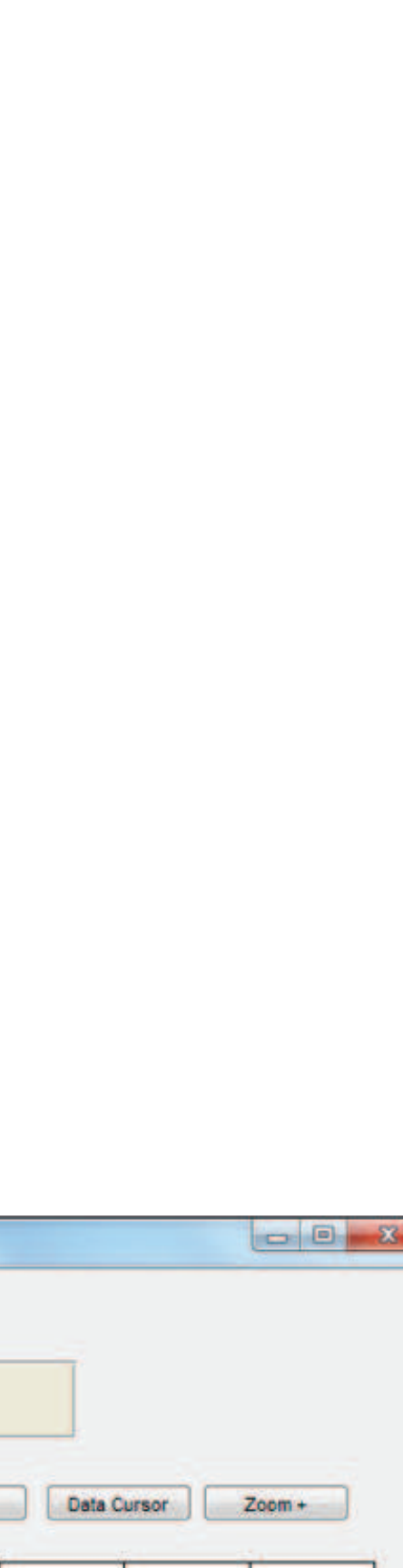


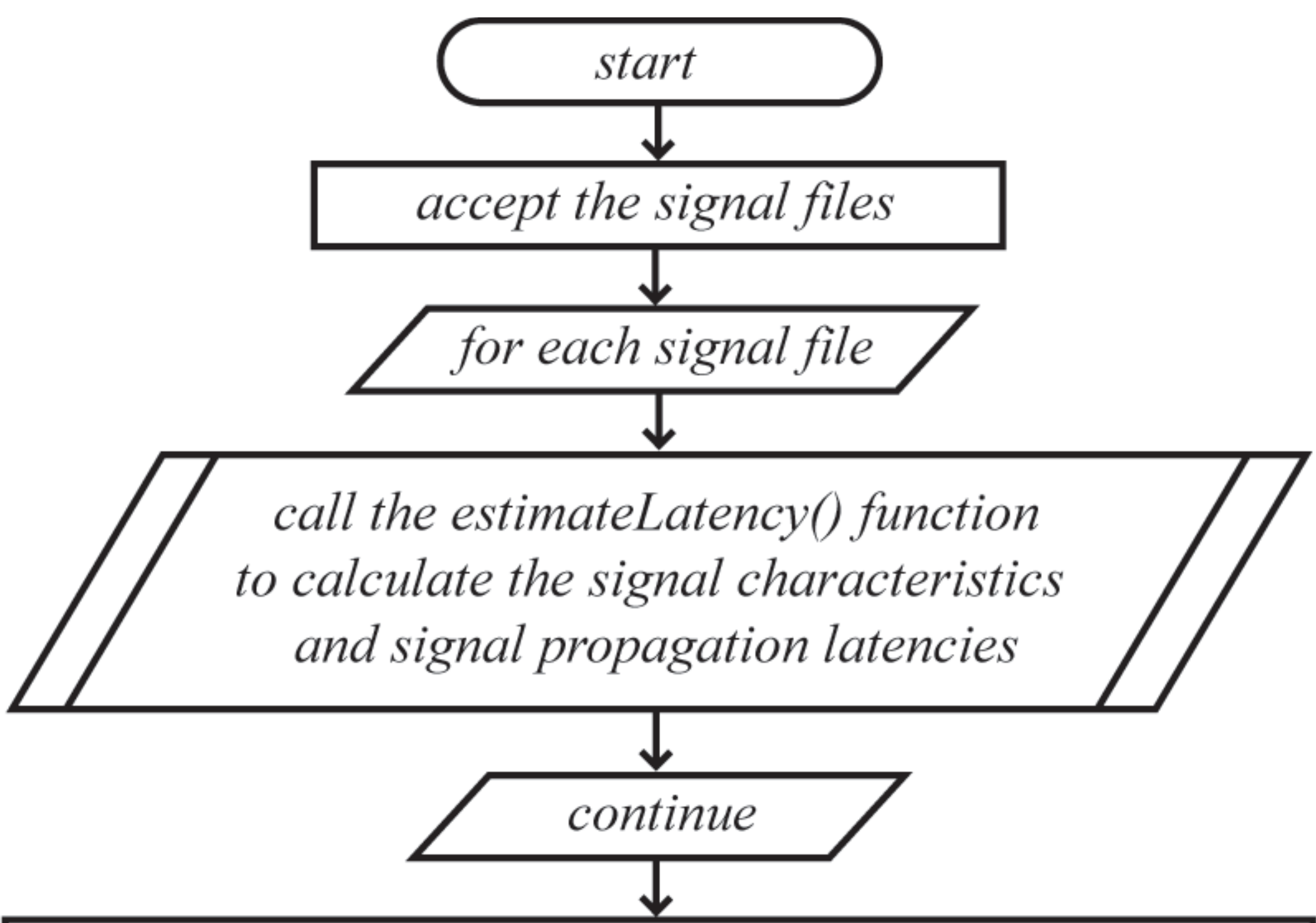

\section{determine the layer based on signal characteristics}

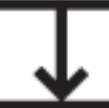

sort the latencies of different layers

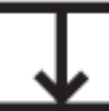

determine the layer activation based on the latencies

Cite as: Mahmud, M., Pasqualotto, E., Bertoldo, A., Girardi, S haschietto, M., Vassanelli, S. An automated method for detection

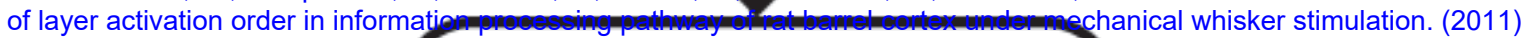
Journal of Neuros cence Methods, 196 (1), pp. 141-150. doi: 10.1016/j.jne Imeth.2010.11.024

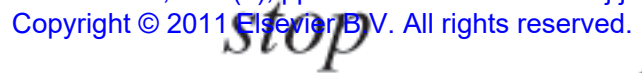




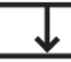

lowpass signal with cutoff frequency $250 \mathrm{~Hz}$

$\downarrow$

determine stimulus start and end time

$\downarrow$

translate signal by setting stimulus-onset to 0

$\downarrow$

determine response-onset

$\downarrow$

translate signal by setting response-onset to 0

calculate signal derivative of the $15 \mathrm{~ms}$

from the response-onset

detect highest peak

down signal direction

antother peak

in $\pm 5 \mathrm{~ms}$ ?

no】

First Event

is absent

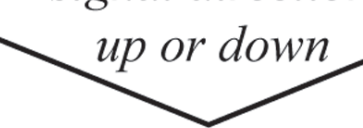

up

detect highest peak

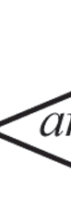

detect this as First Event

is the peak

yes

detect this peak

yes

$\geqslant 10 \mu \mathrm{V}$

\no

First Event

is absent

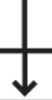

detect the highest negative peak as Second Event

$\downarrow$

detect the highest positive peak in next $100 \mathrm{~ms}$

from the Second Event, this is Third Event

$\downarrow$

detect the highest negative peak in next $200 \mathrm{~ms}$

from the Third Event, this is Fourth Event

$\downarrow$

calculate the latencies of different events-

responseLatency: =responseOnSet-stimulusOnSet

firstEventLatency: =firstEventTime-stimulusOnSet secondEventLatency: = secondEventTime-stimulusOnSet

thirdEventLatency: $=$ thirdEventTime-stimulusOnSet

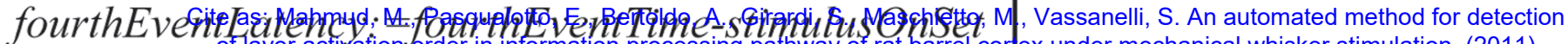
of laver activafion.order in information processing pathwav of ratbarrel con ex under mechanical whisker stimulation. (2011) Journal of Jeuroscience Methods, 196 (1), pp. 141-150. doi: 10.1016/j.jneumeth.2010.11.024 


\section{Current Source Density Analyzer}

Select a Signal source:

Micropiopette Recordings $+$

Select a Directory Containing the *ftt Files of Sinilar Recordings

Selected Directory Path E Un. Vivo Recordings Wipette Recordings

Whisker

Stimi20090715 P37_150gr_mole-analyzedL20090715-New Postion-P37.

IEnow Maloitryt

Cortert of the Directory

Et_x0y02-050_ims $10 \mathrm{~V} 150 \mathrm{~ms}$ dh .

E1 X0y0z-150_ims 10V150msdh

Et_X0y02-250_Ims $10 \mathrm{~V} 150 \mathrm{~ms}$ d.

E1 $\times 0$ y0z-350_-1ms $10 \mathrm{~V} 150 \mathrm{~ms}$ dh

Et_xoy $0 z-450$ - 1 ms $10 \mathrm{~V} 150 \mathrm{~ms}$ d.

E1_xoy0z-550_-1ms 10V150msdh

Et, x0y0z-650_Ims $10 \mathrm{~V} 150 \mathrm{~ms}$ h

Et_x0y0z-750_-1ms 10V150msdi

Et $\times 0 y 0 z-650$ - 1 ms $10 \mathrm{~V} 150 \mathrm{~ms}$ d

E1_x0y0z-950_Ims $10 \mathrm{~V} 150 \mathrm{msd}$

E1_x0y0z-1050_Ims tovisoms

Et_xOyOz-1150_tmistoVi50ms:

Et_x0y0z-1250_t ms 10V150msc

E1 $\times 0 y 0 z-1350-1$ ms $10 \mathrm{~V} 150 \mathrm{msc}$

Et_xoy0z-1450_t ms $10 \mathrm{~V} 150 \mathrm{~ms}$

Et_xoy0z-1550-I ms $10 \mathrm{~V} 150 \mathrm{~ms}$
Et_x0y0z-1650, Ims $10 \mathrm{~V} 150 \mathrm{~ms}$

E1_xoy0z-1750_tmstovisomse

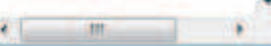

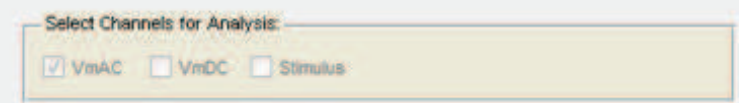

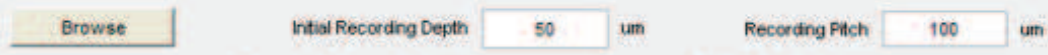

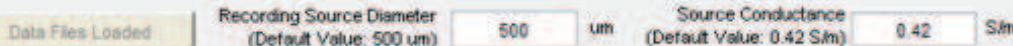

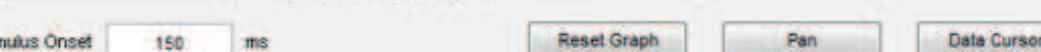

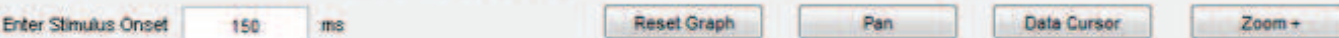

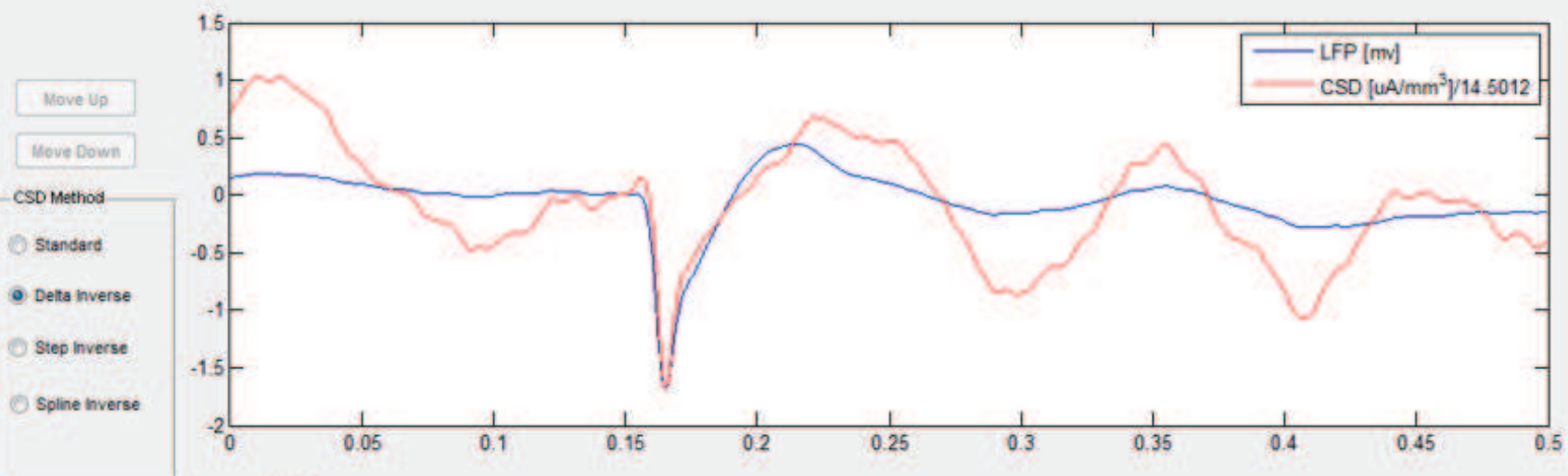

nase

ite as: Mahmud, M., Pasqualotto, E., Bertoldo, A., Girardi, S., Maschietto, M., Vassanelli, S. An automated method for detection

of layer activation order in information processing pathway of rat barrel cortex under mechanical whisker stimulation. (2011) 
Figure 7
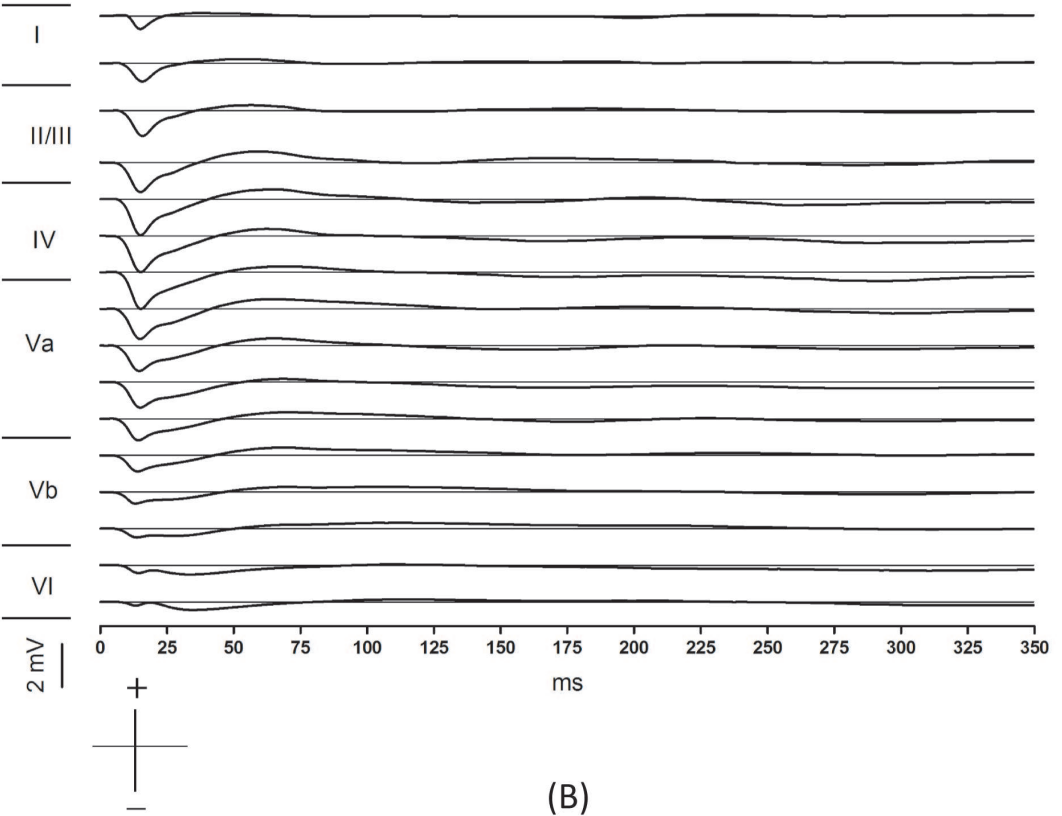

$\mathrm{ms}$

(B)

CSD using Delta iCSD Method

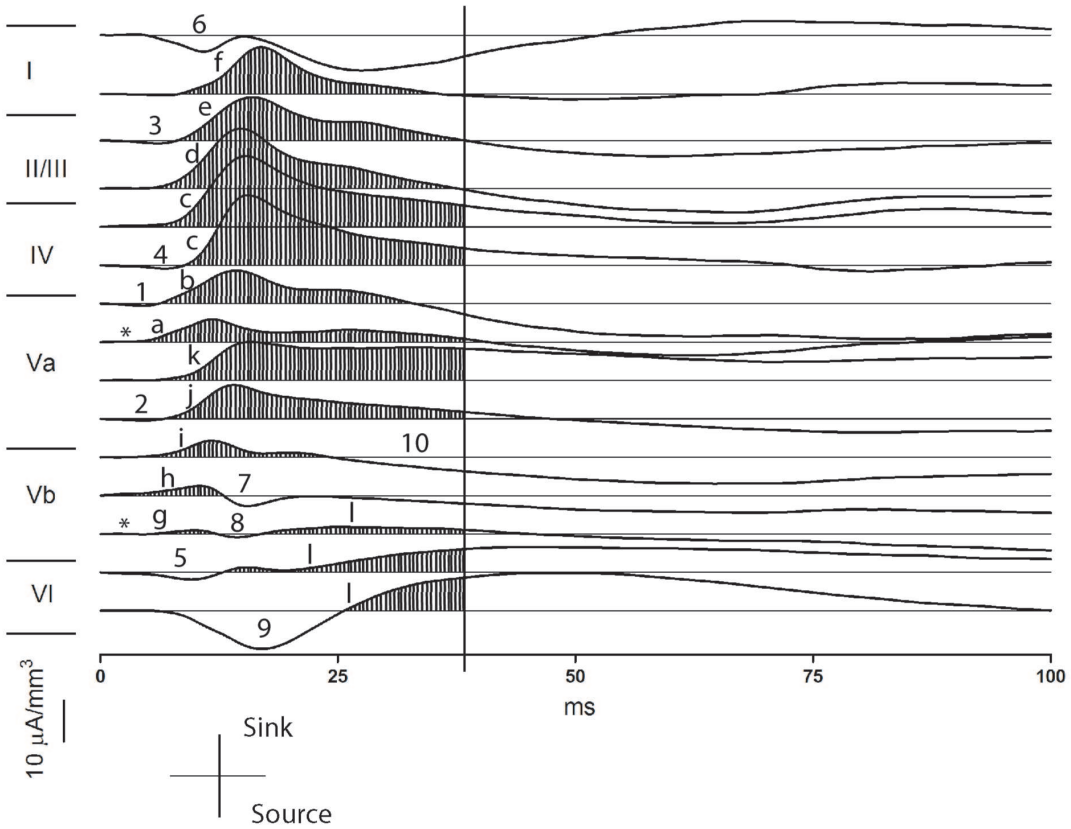

(C)

Cortical Surface

Layer I

Layer II

Layer III

Layer IV

(1)

Layer $\mathrm{T}$

Layer Vb

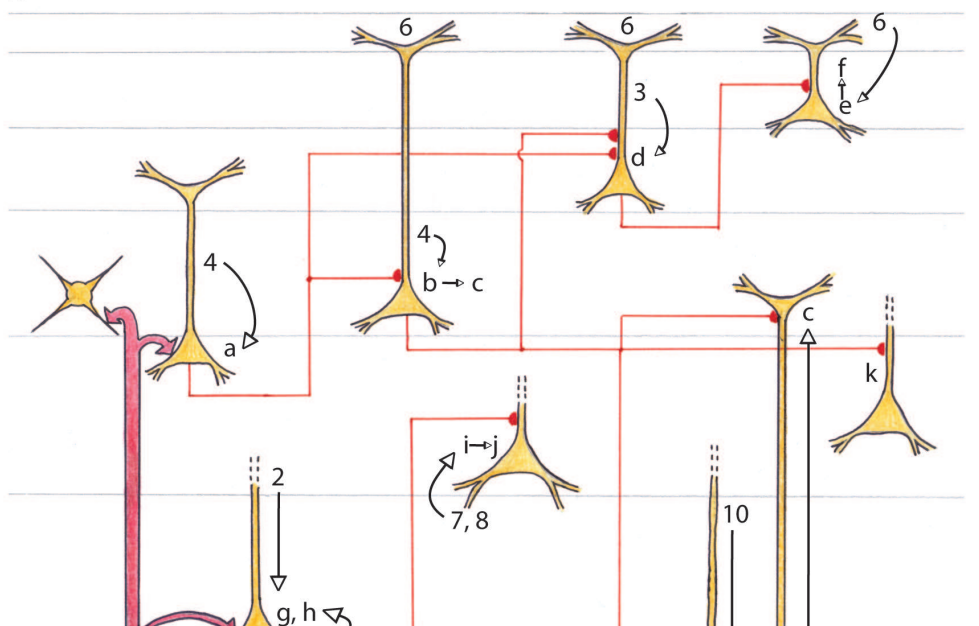

Cite as. Mahmud, M., Pasqualotto, E., Bertold ory., Grardi, S., Maschietto, M., Vassanelli, S. An automated method for detection ofhayer activation order in information processing pathway of rat barrel cortex under mechanical whisker stimulation. (2011) Journal of Neuroscience Methrds, 196 (1), pp. 141-150. doi: 10.1016/j.jneumeth.2010.11.024

Copyrighto 2011 Elsevier B.V. All rights reserved. 
Figure 8

\section{Barrel Cortex Architecture}

Cortical Surface

Layer I

Layer II

Layer III

Layer Va

Layer IV

Layer VI

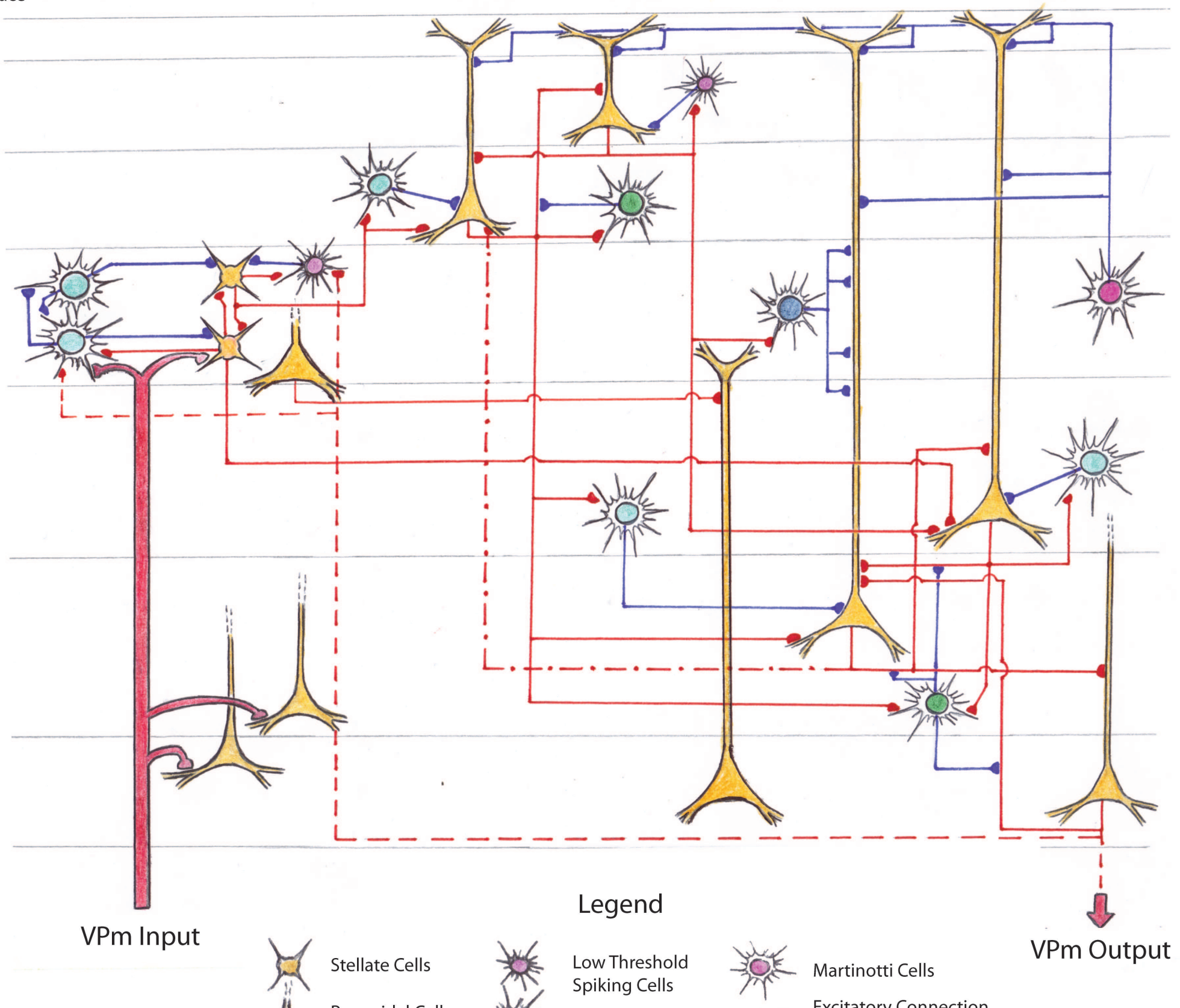

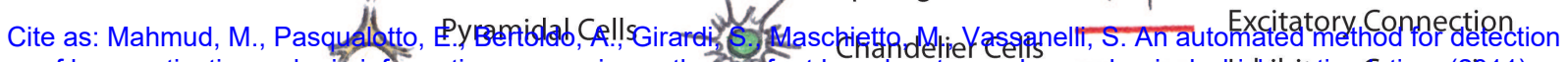

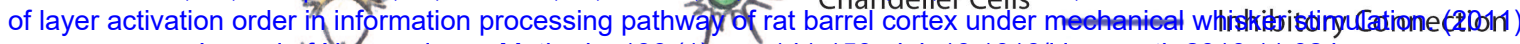
Journal of Nerurescience Methods, 196 (1) pp. 141-150. doi: 10.1016/j.jnezumeth.2010.11.024

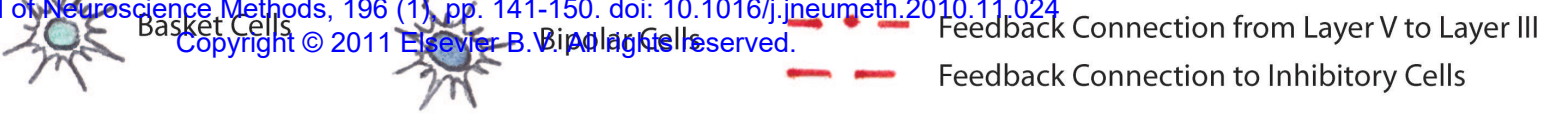




\section{Latencies Calculated using LFP and CSD}

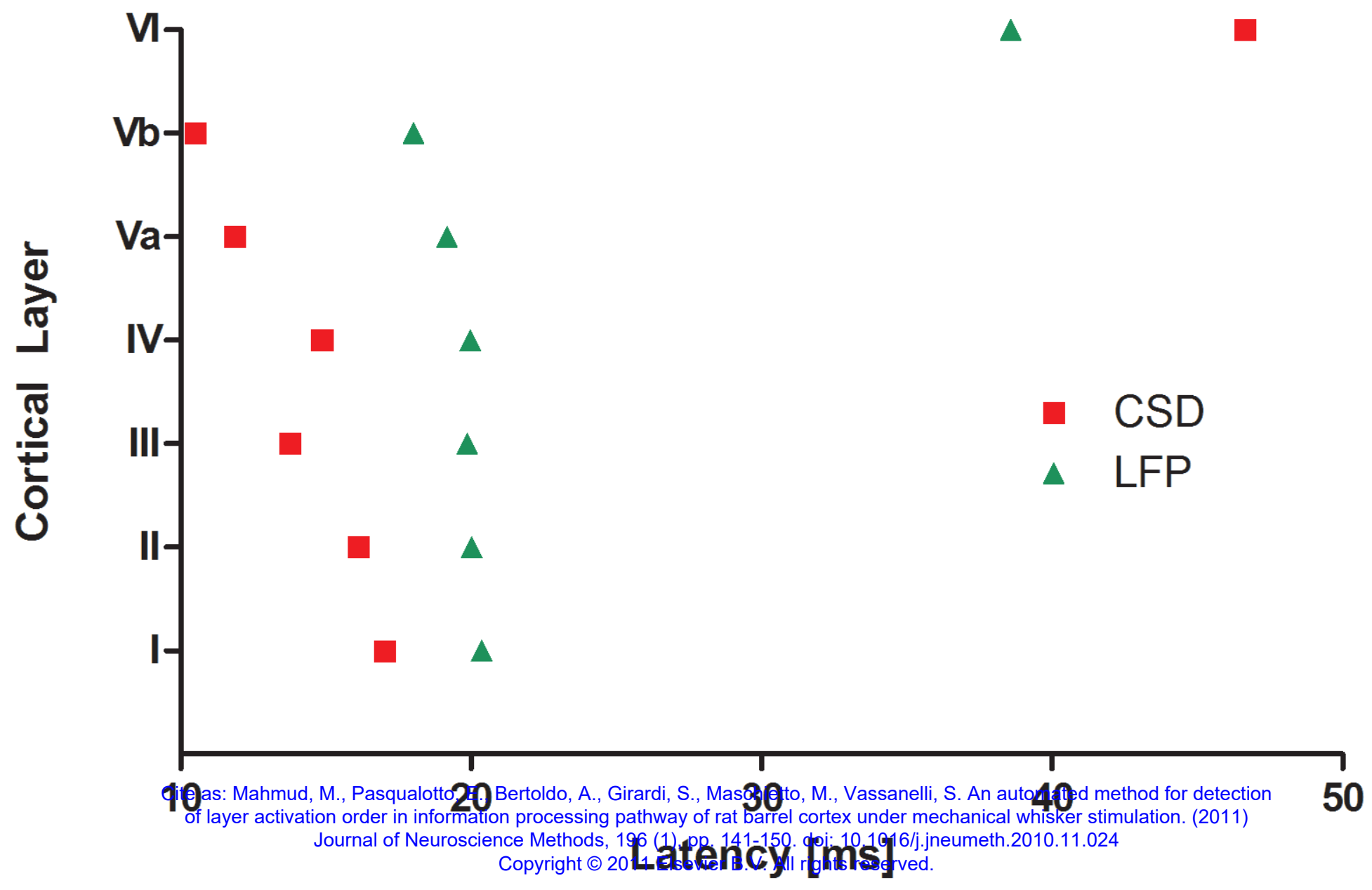




\section{Layer Activation Order with LFP}

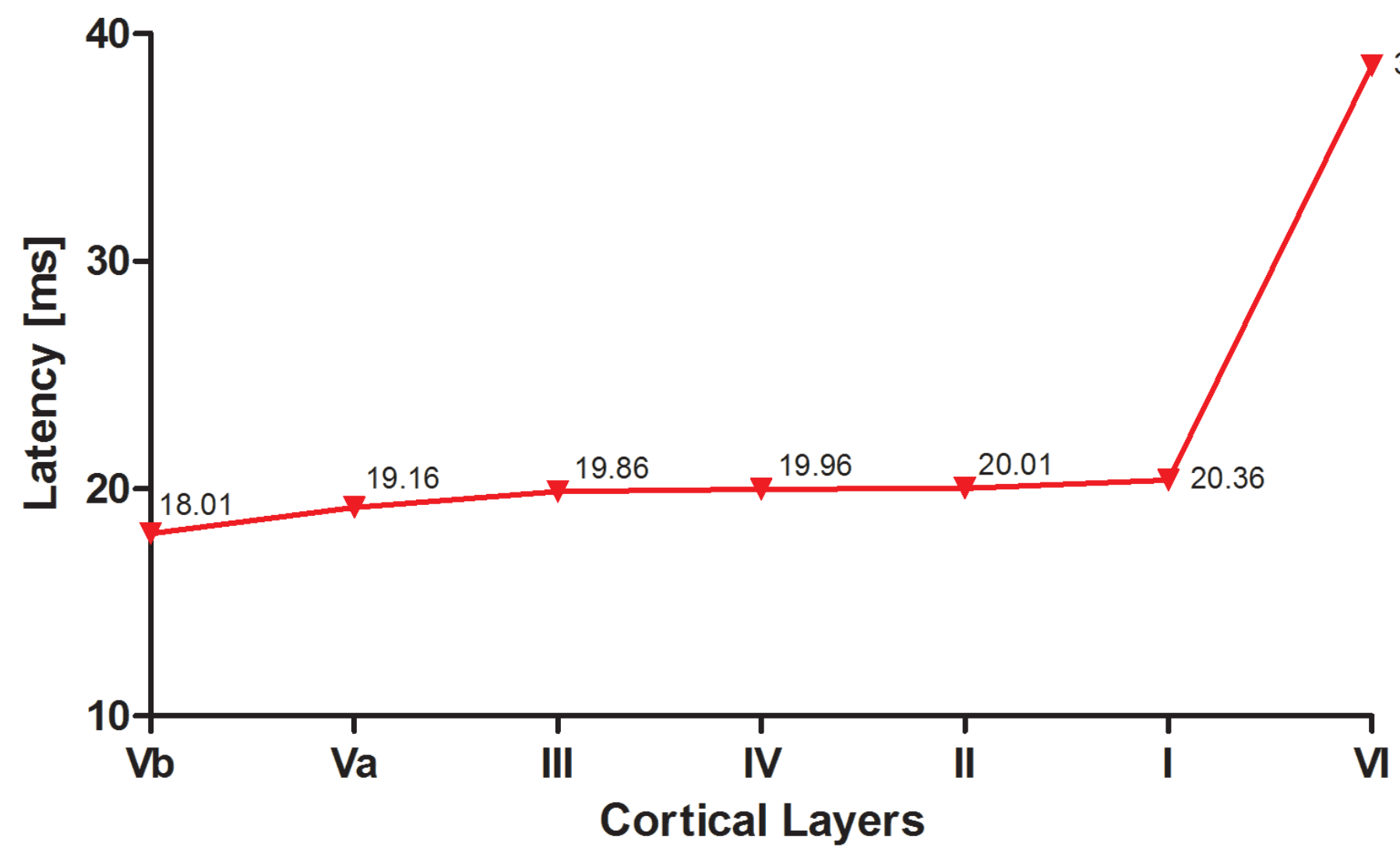

\section{Layer Activation Order with CSD}

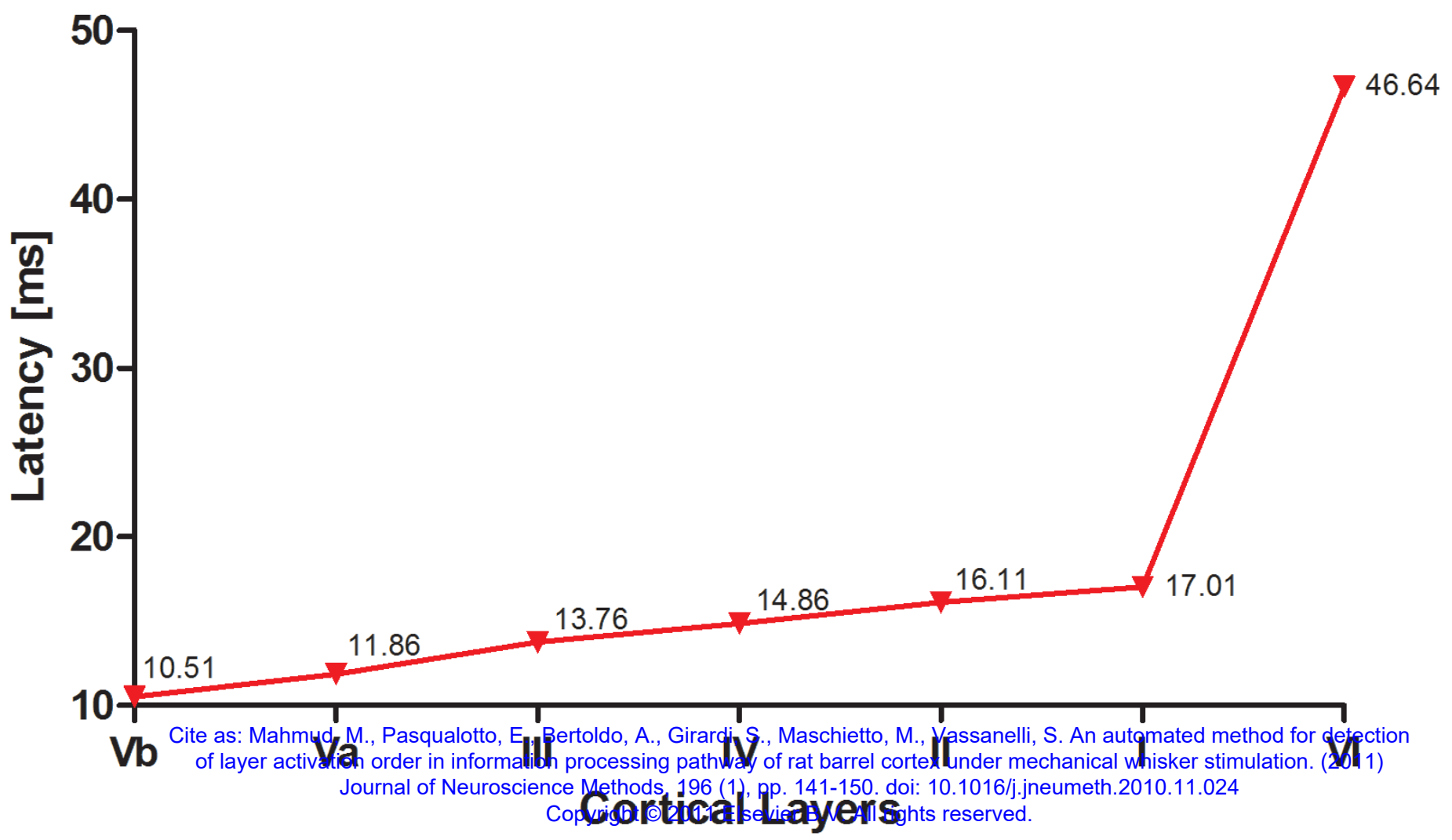




\section{Comparison of Barrel Cortex Layer Activation}

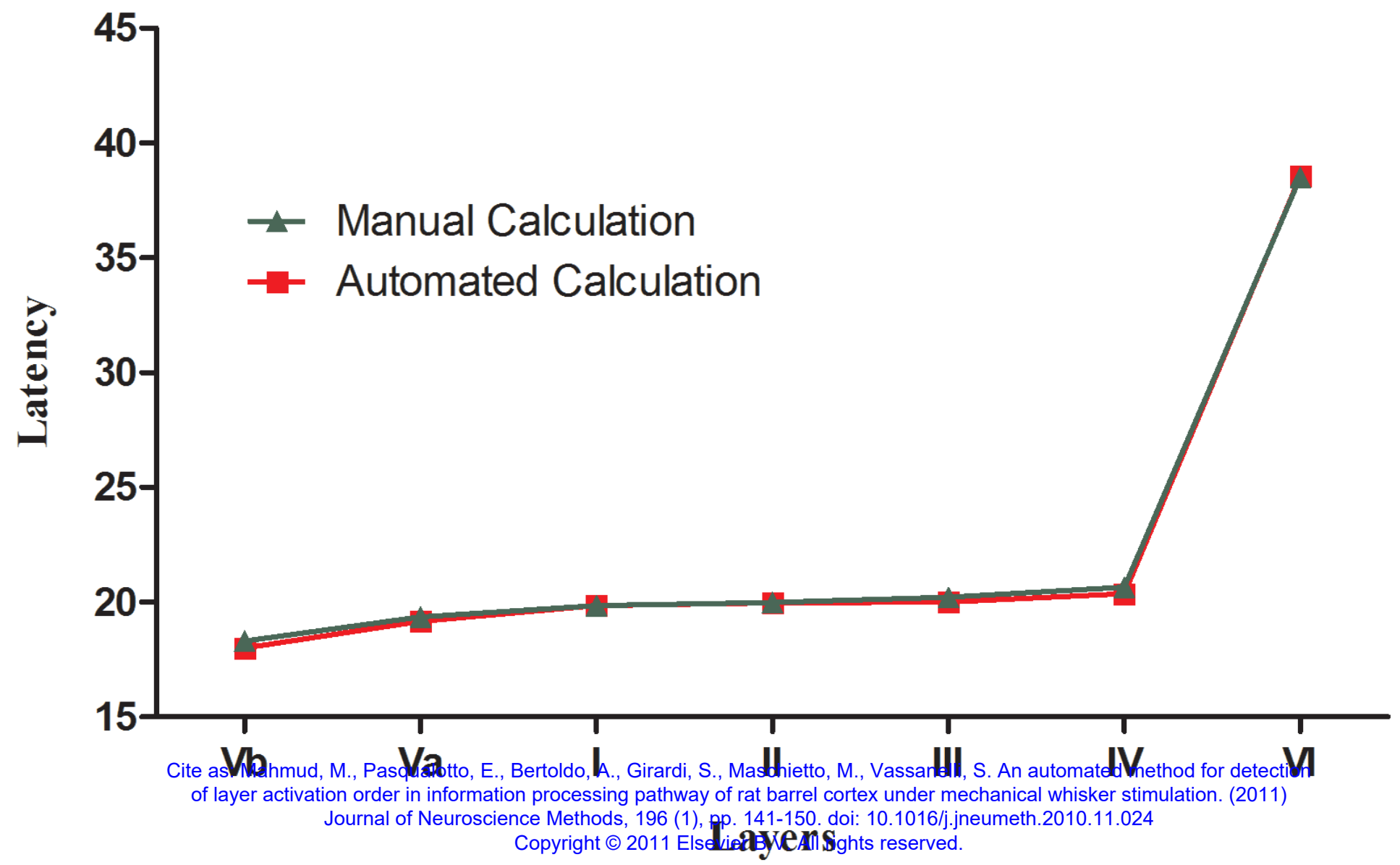




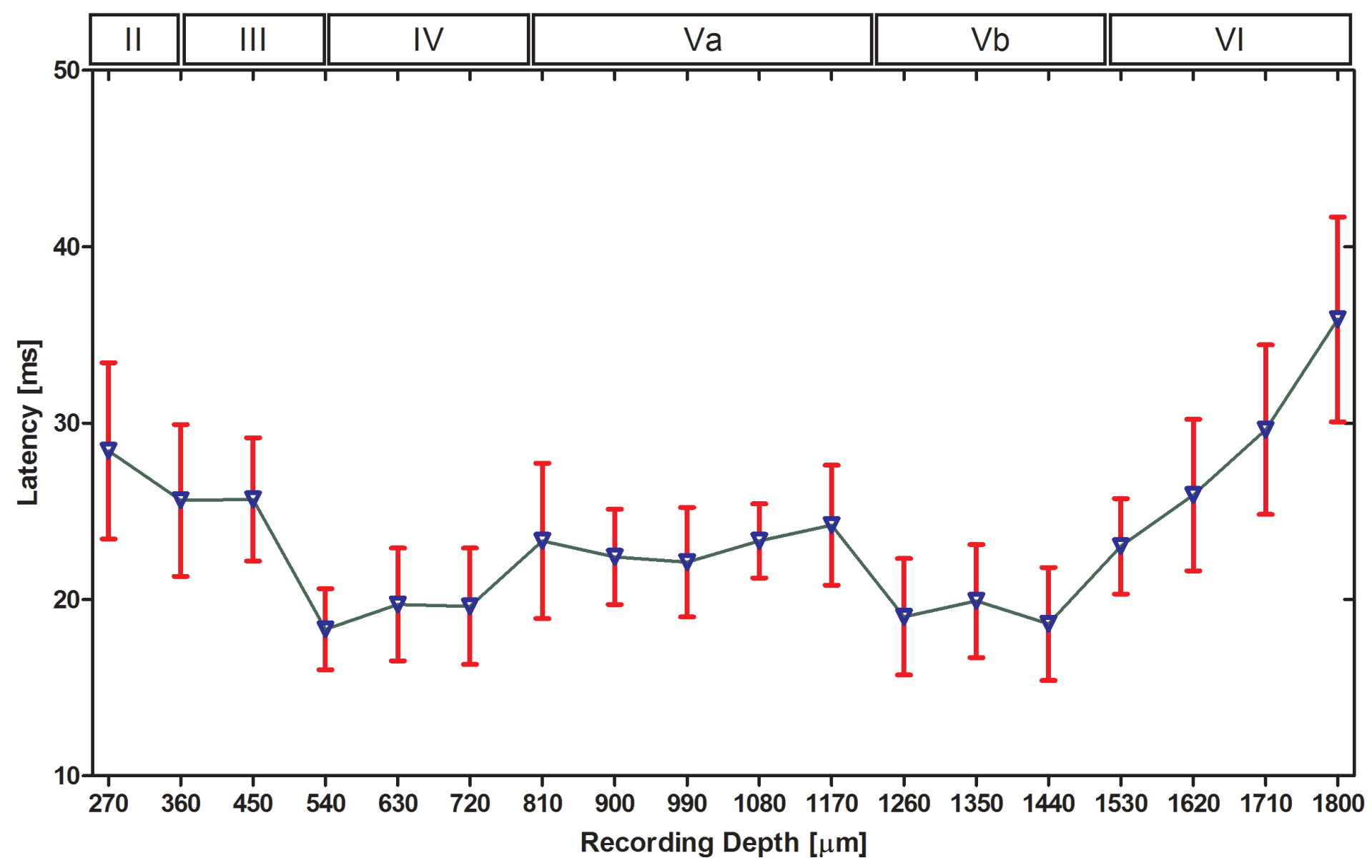

\section{Latency in CSD Profile Obtained from Grand Average LFP Profile}

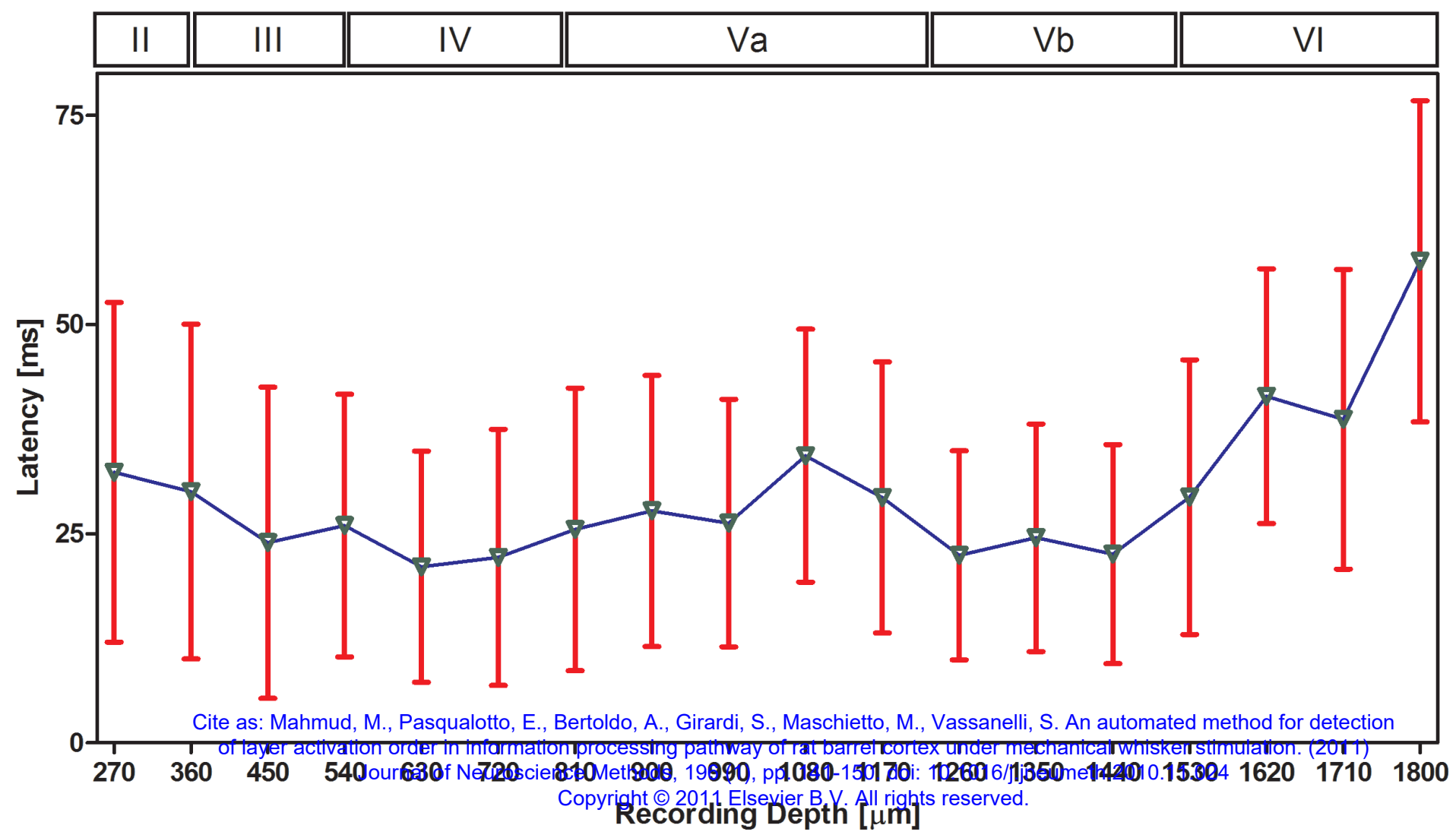

